\title{
ON THE ROLE OF SHARP CHAINS IN THE TRANSPORT THEOREM
}

\author{
L. FALACH AND R. SEGEV
}

\begin{abstract}
A generalized transport theorem for convecting irregular domains is presented in the setting of Federer's geometric measure theory. A prototypical $r$-dimensional domain is viewed as a flat $r$-chain of finite mass in an open set of an $n$-dimensional Euclidean space. The evolution of such a generalized domain in time is assumed to be in accordance to a bi-Lipschitz type map. The induced curve is shown to be continuous with respect to the flat norm and differential with respect to the sharp norm on currents in $\mathbb{R}^{n}$. A time dependent property is naturally assigned to the evolving region via the action of an $r$-cochain on the current associated with the domain. Applying a representation theorem for cochains the properties are shown to be locally represented by an $r$-form. Using these notions a generalized transport theorm is presented.
\end{abstract}

\section{INTRODUCTION}

Reynolds' transport theorem [25], offers a general form for the formulation of basic conservation laws in continuum mechanics and in particular in fluid dynamics. The traditional formulation of Reynolds theorem (or LeibnizReynolds theorem) deals with the time derivative of the integral of $\omega(t)$, a time dependent scalar field, over a time evolving spatial region $\mathcal{P}(t)$ in a Euclidean physical space. The region $\mathcal{P}(t)$ is assumed to be the image of a domain under a smooth motion, where the domain is assumed to be sufficiently regular such that the classical divergence theorem is applicable (for example a Lipschitz domain). The transport theorem states that

$$
\left.\frac{d}{d t}\left(\int_{\mathcal{P}(t)} \omega(t) d L^{n}\right)\right|_{t=\tau}=\left.\int_{\mathcal{P}(\tau)} \frac{\partial \omega}{\partial t}\right|_{t=\tau} d L^{n}+\int_{\partial \mathcal{P}(\tau)} \omega(\tau) V \cdot \nu d H^{n-1},
$$

with $\nu$, the unit exterior normal to the boundary $\partial \mathcal{P}$ and $V$ the velocity associated with the smooth motion. It is noted that the proof of the Reynolds' transport theorem is attributed by Truesdell and Toupin [29, p. 347] to Spielrein (1916).

In the study of deforming thin films or evolving phase boundary a transport relation for surface integrals is of interest. Such a theorem is usually refereed to as Surface Transport Theorem. It seems that the basic notions of surface transport theorem in the setting of continuum mechanics were first introduced in [15] with the introduction of the surface divergence operator. 
Betounes [3], examined the kinematics of an $r$-dimentional submanifold embedded in an $n$-dimentional semi-Riemannian manifold. Betounes's formulation brings to light the strong dependence of the formulation of the surface transport theorem on the availability of the mean curvature normal. In [22] Gurtin et al. formulated a surface transport theorem for moving interfaces while additional study and applications were presented in [12, 2, 16, 13, 14, to name a few.

In the aforementioned versions of the transport theorem, the regularity of the evolving domain is tacitly assumed. The inclusion of irregular domains, where such notions as the exterior normal and mean curvature normal not applicable, in the formulation of the transport theorem has been presented recently in [27, 28]. Seguin \& Fried construct a generalized transport theorem using the setting of Harrison's theory of differential chains (see [18, 17]). The proposed formulation allows for singularities to evolve in the domains, e.g., the domains may develop holes, split into pieces and the fractal dimensions associated with the domain considered may change. As their formulation of a transport theorem relies of a dual relation between the domains and the properties considered the resulting representation for theses properties is fairly regular.

In [7, a transport theorem is presented in the setting of general manifolds. The domain of integration considered is viewed as a de-Rham current of compact support thus including highly irregular domains. The domain is assumed to evolve under a smooth map and integration of a given property in the classical theory is replaced with the action of the evolving current on a smooth differential form.

In the present work we wish to present a version of the transport theorem in the setting of Federer's geometric measure theory. A generalized domain, or a control volume, is viewed as a flat $r$-chain of finite mass $T$. The current $T$ is assumed to evolve under the action of $\kappa$, a time dependent bi-Lipschitz homeomorphisms. With $\mathcal{I} \subset \mathbb{R}$ representing a time interval the control volume at time $t \in \mathcal{I}$ is represented by the flat $r$-chain $\kappa_{t \#} T$, given by the pushforward of $T$ by $\kappa_{t}=\kappa(t)$. A considerable portion of this work is dedicated to the study of the properties of the induced curve $t \mapsto \kappa_{t \#} T$ which is shown to be continuous with respect to the flat topology of currents and differentiable with respect to the sharp topology of currents. The integration of a given property over the domain is generalized to the action of an $r$ cochain on the current.

It is observed that Lipschitz continuity arises naturally as a characteristic of the proposed setting both is the resulting representation of properties, by sharp forms, and in the regularity of the motion, independently. 


\section{Notation ANd Preliminaries}

In this section we review some of the fundamental concepts of the theory of currents in an $n$-dimensional Euclidean space. Throughout, the notation is in the same spirit of [8, Chapter 4].

Let $U$ be an open set in $\mathbb{R}^{n}$, the notation $\mathfrak{D}^{r}(U)$ is used for the vector space of smooth, compactly supported real valued differential $r$-forms defined on $U$. The vector space $\mathfrak{D}^{r}(U)$ is endowed with a family of semi-norms $\|\cdot\|_{i, K}$ such that for a compact $K \subset U$ and $i \in \mathbb{N}$,

$$
\|\phi\|_{i, K}=\sup \left\{\left\|D^{j} \phi(x)\right\| \mid x \in K, 0 \leq j \leq i\right\} .
$$

(Here, the norm $\left\|D^{j} \phi(x)\right\|$ is induced by some norm on tensors in $\mathbb{R}^{n}$.) This family of seminorms endows $\mathfrak{D}^{r}(U)$ with a locally convex topology. For $\phi \in \mathfrak{D}^{r}(U)$ we use $d \phi$ to denote the exterior derivative of $\phi$, an element of $\mathfrak{D}^{r+1}(U)$. A linear functional $T: \mathfrak{D}^{r}(U) \rightarrow \mathbb{R}$ continuous with respect to the topology on $\mathfrak{D}^{r}(U)$ is referred to as an $r$-dimensional de Rham current in $U$. The collection of all $r$-dimensional currents defined on $U$ forms the vector space $\mathfrak{D}_{r}(U)=\left[\mathfrak{D}^{r}(U)\right]^{*}$, the dual vector space of $\mathfrak{D}^{r}(U)$. Let $T \in \mathfrak{D}_{r}(U)$ with $r \geq 1$ then, $\partial T$, the boundary of $T$, is the element of $\mathfrak{D}_{r-1}(U)$ defined by

$$
\partial T(\phi)=T(d \phi), \quad \text { for all } \quad \phi \in \mathfrak{D}^{r-1}(U) .
$$

Thus, we have the boundary operator $\partial=d^{*}$, the adjoint operator of the exterior derivative. The support of a current $T \in \mathfrak{D}_{r}(U)$ is defined by

$$
\operatorname{spt}(T)=U \backslash \bigcup W,
$$

where each $W$ is an open subset of $U$ such that $T(\phi)=0$ for all $\phi \in \mathfrak{D}^{r}(U)$, with $\operatorname{spt}(\phi) \subset W$. Generally speaking, the support of a current $T \in \mathfrak{D}_{r}(U)$ need not be compact, however, in this work all the currents considered will be of compact support.

The inner product in $\mathbb{R}^{n}$ induces an inner product in $\bigwedge_{r} \mathbb{R}^{n}$, the vector space of $r$-vector in $\mathbb{R}^{n}$, and $|\xi|$ will denote the resulting norm of an $r$-vector $\xi$. An $r$-vector $\xi$ may be written by $\xi=\sum_{\lambda \in \Lambda(r, n)} \xi^{\lambda} e_{\lambda}$ where $\Lambda(r, n)$ is the collection of increasing maps from $\{1, \ldots, r\}$ to $\{1, \ldots, n\}$, and $\left\{e_{\lambda}\right\}$ is the standard basis for $\bigwedge_{r} \mathbb{R}^{n}$ defined by $e_{\lambda}=e_{\lambda(1)} \wedge \cdots \wedge e_{\lambda(r)}$. Thus, with the above notation, $|\xi|=\sqrt{\langle\xi, \xi\rangle}=\sqrt{\left(\xi^{\lambda}\right)^{2}}$. Given $\phi \in \mathfrak{D}^{r}(U)$, for every $x \in U$, $\phi(x)$ is an $r$-covector. For $\phi(x)$, as well as any other covector, one defines

$$
\|\phi(x)\|_{0}=\sup \{\phi(x)(\xi)|| \xi \mid \leq 1, \xi \text { is a simple } r \text {-vector }\} \text {. }
$$

For a compact subset $K \subset U$, define the $K$-Lipschitz constant of $\phi \in \mathfrak{D}^{r}(U)$ by

$$
\mathfrak{L}_{\phi, K}=\sup _{x, y \in K} \frac{\|\phi(y)-\phi(x)\|_{0}}{|y-x|} .
$$

In addition to the topology of test functions, three additional topologies will be examined on $\mathfrak{D}^{r}(U)$ each of which is induced by a corresponding family of 
semi-norms. For $K \subset U$ the $K$-comass semi-norm of $\phi \in \mathfrak{D}^{r}(U)$ is defined by

$$
M_{K}(\phi)=\operatorname{ess} \sup _{x \in K}\left\{\|\phi(x)\|_{0}\right\},
$$

the $K$-flat semi-norm on $\mathfrak{D}^{r}(U)$ is defined by

$$
F_{K}(\phi)=\operatorname{ess} \sup _{x \in K}\left\{\|\phi(x)\|_{0},\|d \phi(x)\|_{0}\right\},
$$

and the $K$-sharp semi-norm on $\mathfrak{D}^{r}(U)$ is defined by

$$
S_{K}(\phi)=\sup \left\{\sup _{x \in K}\|\phi(x)\|_{0},(r+1) \mathfrak{L}_{\phi, K}\right\} .
$$

The factor $(r+1)$ in the above definition is introduced so that

$$
F_{K}(\phi) \leq S_{K}(\phi), \quad \text { for all } \phi \in \mathfrak{D}^{r}(U) .
$$

(The essential supremum is used above in spite of the fact that we consider smooth functions because we are going to apply below these definitions to essentially bounded functions.) Later on, when the ess sup in each of the above terms is evaluated over $U$, we shall write $M(\phi), F(\phi), S(\phi)$ for the mass, flat and Sharp norms, respectively.

For $T \in \mathfrak{D}_{r}(U)$, the mass of $T$ is dually defined by

$$
M(T)=\sup \left\{T(\phi) \mid \phi \in \mathfrak{D}^{r}(U), M(\phi) \leq 1\right\},
$$

and

$$
M_{K}(T)=\sup \left\{T(\phi) \mid \phi \in \mathfrak{D}^{r}(U), M_{K}(\phi) \leq 1\right\} .
$$

An $r$-dimensional current $T$ is said to be represented by integration if there exists a Radon measure $\mu_{T}$ and an $r$-vector valued, $\mu_{T}$-measurable function, $\vec{T}$, with $|\vec{T}(x)|=1$ for $\mu_{T}$-almost all $x \in U$, such that

$$
T(\phi)=\int_{U} \phi(\vec{T}) d \mu_{T}, \quad \text { for all } \quad \phi \in \mathfrak{D}^{r}(U) .
$$

A sufficient condition for an $r$-dimensional current, $T$, to be represented by integration is that $T$ is a current of locally finite mass, i.e., $M_{K}(T)<\infty$ for all compact subsets $K \subset U$. An $r$-current $T$ of compact support is said to be a normal current if both $T$ and $\partial T$ are represented by integration. The notion of normal currents leads to the following definition

$$
N(T)=M(T)+M(\partial T),
$$

and clearly, every $T \in \mathfrak{D}_{r}(U)$ such that $N(T)<\infty$ is a normal $r$-current. The vector space of all $r$-dimensional normal currents in $U$ is denoted by $N_{r}(U)$ and for a compact set $K$ of $U$,

$$
N_{r, K}(U)=N_{r}(U) \cap\{T \mid \operatorname{spt}(T) \subset K\} .
$$

The $K$-flat norm on $\mathfrak{D}_{r}(U)$ is given by

$$
F_{K}(T)=\sup \left\{T(\phi) \mid F_{K}(\phi) \leq 1\right\} .
$$


It follows naturally from the foregoing definition that

$$
F_{K}(\partial T) \leq F_{K}(T) .
$$

Note that if $T \in \mathfrak{D}_{r}(U)$ such that $F_{K}(T)<\infty$, then, $\operatorname{spt}(T) \subset K$. For a given compact subset $K \subset U$, the set $F_{r, K}(U)$ is defined as the $F_{K}$-closure of $N_{r, K}(U)$ in $\mathfrak{D}_{r}(U)$. In addition, set

$$
F_{r}(U)=\bigcup_{K} F_{r, K}(U)
$$

where the union is taken over all compact subsets $K$ of $U$. An element in $F_{r}(U)$ is referred to as a flat $r$-chain in $U$.

For $T \in F_{r, K}(U)$ it can be shown that $F_{K}(T)$ is given by

$$
F_{K}(T)=\inf \left\{M(T-\partial S)+M(S) \mid S \in \mathfrak{D}_{r+1}(U), \operatorname{spt}(S) \subset K\right\},
$$

and by taking $S=0$ it follows that

$$
F_{K}(T) \leq M(T) \text {. }
$$

In addition, any element $T \in F_{r, K}(U)$ may be represented by $T=R+\partial S$ where $R \in \mathfrak{D}_{r}(U), S \in \mathfrak{D}_{r+1}(U)$, such that $\operatorname{spt}(R) \subset K, \operatorname{spt}(S) \subset K$, and

$$
F_{K}(T)=M(R)+M(S),
$$

so that $R$ and $S$ are of finite mass. By Equation (2.14) we note that the boundary of a flat $r$-chain is a flat $(r-1)$-chain.

The following representation theorem for flat chains is given in $[8$, Section 4.1.18]. Let $T$ be a flat $r$-chain in $U$, then, $T$ is represented by

$$
T=L^{n} \wedge \eta+\partial\left(L^{n} \wedge \xi\right)
$$

with $\eta$ an $L^{n}\left\llcorner U\right.$-summable $r$-vector field and $\xi$ an $L^{n}\llcorner U$-summable $(r+1)$ vector field $\xi$. Here, $L^{n}\llcorner U$ denotes the restriction of the $n$-dimensional Lebesgue measure to $U$ and for any $p$-current $T$ and any $r$-vector field $\eta$, the $(p+r)$-current $T \wedge \eta$ is given by

$$
T \wedge \eta(\psi)=T(\psi\llcorner\eta) .
$$

For $\phi \in \mathfrak{D}_{r}(U)$, the action $T(\phi)$ is given therefore by

$$
T(\phi)=\int_{U}(\phi(\eta)+d \phi(\xi)) d L^{n} .
$$

A real valued linear functional $X$ defined on $F_{r}(U)$ is said to be a flat $r$ cochain in $U$ if there exists a number $c<\infty$ such that for any $K \subset U$ compact subset

$$
X(T) \leq c F_{K}(T), \quad \text { for all } T \in F_{r, K}(U) .
$$

The infimum of all bounds $c$ is the norm of $X$. An $r$-form $\omega$ in $U$ is said to be a flat $r$-form in $U$ if $\omega$ and $d \omega$, taken in the distributional sense, are $L^{n}$ measurable and essentially bounded (see [19, p. 38]). That is, a measurable $r$-form $\omega$ is a flat $r$-form in $U$ if and only if $F(\omega)<\infty$. An important result, Wolfe's representation theorem (see [30, ch. 7], [8, sec. 4.1.19]), states that 
each flat $r$-cochains $X$ can be isometrically identified with a flat $r$-form in $U$.

The notion of sharp chains was initially introduced in Whitney's classical monograph [30]. In the following we present a construction in the spirit of the formulation of the theory of flat chains in [8] (who does not consider sharp chains).

The $K$-sharp norm on $\mathfrak{D}_{r}(U)$ is given by

$$
S_{K}(T)=\sup \left\{T(\phi) \mid S_{K}(\phi) \leq 1\right\} .
$$

For a given compact subset $K \subset U$, the set $S_{r, K}(U)$ is defined as the $S_{K^{-}}$ closure of $N_{r, K}(U)$ in $\mathfrak{D}_{r}(U)$. In addition, we set

$$
S_{r}(U)=\bigcup_{K} S_{r, K}(U),
$$

where the union is taken over all compact subsets $K$ of $U$. An element in $S_{r}(U)$ is referred to as a sharp r-chain in $U$. Note that as $F_{r, K}(U) \subset S_{r, K}(U)$ and the set $N_{r, K}(U)$ is a dense set in both $F_{r, K}(U)$ and $S_{r, K}(U)$, it follows that every sharp $r$-chain may be viewed a the limit, in the sharp topology, of a sequence of flat $r$-chains. A representation theorem for general sharp chains is beyond the scope of this work, however, for sharp chains of finite mass such a representation theorem may be found in [30, Chapter. XI].

Let $X$ be a real valued linear functional defined on $S_{r}(U)$, then, $X$ is said to be a sharp $r$-cochain in $U$ provided there exists a number $b<\infty$ such that for any $K \subset U$ compact subset

$$
X(T)<b S_{K}(T), \quad \text { for all } T \in S_{r, K}(U) .
$$

The infimum of all bounds $b$ is the norm of $X$. An $r$-form $\omega$ in $U$ is said to be a sharp $r$-form in $U$ if the coefficients of $\omega$ are bounded and Lipschitz continuous, i.e., $\omega$ is a sharp $r$-from if and only if

$$
S(\omega)=\sup \left\{\sup _{x \in U}\|\omega(x)\|_{0},(r+1) \mathfrak{L}_{\omega}\right\}<\infty .
$$

Theorem 2.1. Let $X$ be a sharp $r$-cochain in $\mathbb{R}^{n}$. Then, $X$ can be isometrically identified with $D_{X}$, a sharp $r$-form in $\mathbb{R}^{n}$. That is, for any flat chain given by $T=L^{n} \wedge \eta+\partial\left(L^{n} \wedge \xi\right)$

$$
X(T)=\int\left[D_{X}(\eta)+d D_{X}(\xi)\right] d L^{n} .
$$

For a sharp $r$-chain, $T$, as flat chains are dense in the space of sharp chains we have $T=\lim _{i \rightarrow \infty}^{S} T_{i}$ and

$$
X(T)=\lim _{i \rightarrow \infty} X\left(T_{i}\right) .
$$

For the proof see [30, Section V.10]

Let $U \subset \mathbb{R}^{n}, V \subset \mathbb{R}^{m}$ be open sets and let $T \in \mathfrak{D}_{k}(U), S \in \mathfrak{D}_{l}(V)$. Then, the Cartesian product of $T$ and $S$ is an element of $\mathfrak{D}_{k+l}(U \times V)$ denoted by 
$T \times S$ and defined as follows. Let $\omega \in \mathfrak{D}^{k+l}(U \times V)$ be given by

$$
\omega=\sum_{|\alpha|=k,|\beta|=l} \omega_{\alpha \beta}(x, y) d x^{\alpha} \wedge d y^{\beta},
$$

where $x \in U, y \in V$, and $\alpha, \beta$ are multi-indices. Then,

$$
T \times S(\omega)=T\left(\sum_{|\alpha|=k} S\left(\sum_{|\beta|=l} \omega_{\alpha \beta}(x, y) d y^{\beta}\right) d x^{\alpha}\right) .
$$

For the properties of the Cartesian products of currents see [10, Section 2.3]. Let $U \subset \mathbb{R}$, then, the line segment $[a, b] \subset U$ defines naturally an element of $\mathfrak{D}_{1}(U)$ such that for $\phi \in \mathfrak{D}_{1}(V)=C_{0}^{\infty}(V)$ the action $[a, b](\phi)$ is $[a, b](\phi)=\int_{a}^{b} \phi(t) d L_{t}^{1}$. Consider the case of $T \in \mathfrak{D}_{r}(U)$, then, a given $\omega \in \mathfrak{D}^{r+1}(V \times U)$ may be split into a horizontal and a vertical component in the form $\omega=\omega_{H}+\omega_{V}$ by

$$
\omega_{H}(t, x)=\sum_{|\alpha|=r} \omega_{H \alpha}(t, x) d t \wedge d x^{\alpha}, \quad \omega_{V}(t, x)=\sum_{|\alpha|=r+1} \omega_{V \alpha}(t, x) d x^{\alpha},
$$

where, $e_{t}$ is the pre-dual of $d t$. It is observed that

$$
\sum_{|\alpha|=r} \omega_{H \alpha}(t, x) d x^{\alpha}=\omega\left\llcorner e_{t} .\right.
$$

Applying Equation (2.23) to $([a, b] \times T)(\omega)$,

$$
\left.([a, b] \times T)(\omega)=\int_{a}^{b} T\left(\omega_{H}\right) d L_{t}^{1}=\int_{a}^{b} T\left(e_{t}\right\lrcorner \omega\right) d L_{t}^{1}=\int_{a}^{b} e_{t} \wedge T(\omega) d L_{t}^{1} .
$$

Generally speaking, the Cartesian product of two flat chains is not a flat chain. However the Cartesian product of a flat chain and a normal current is a flat chain (see [8, Sec. 4.1.12].

\section{LIPSCHITZ MAPS}

In this section we briefly review some of the relevant properties of Lipschitz mappings. From the point of view of kinematics, Lipschitz mappings will be used to model the evolution of body-like regions in space.

A map $\mathcal{F}: U \rightarrow V$ from an open set $U \subset \mathbb{R}^{n}$ to an open set $V \subset \mathbb{R}^{m}$, is said to be a (globally) Lipschitz map if there exists a number $c<\infty$ such that $|\mathcal{F}(x)-\mathcal{F}(y)| \leq c|x-y|$ for all $x, y \in U$. The Lipschitz constant of $\mathcal{F}$ is defined by

$$
\mathfrak{L}_{\mathcal{F}}=\sup _{x, y \in U} \frac{|\mathcal{F}(y)-\mathcal{F}(x)|}{|y-x|} .
$$

The map $\mathcal{F}: U \rightarrow V$ is said to be locally Lipschitz if for every $x \in U$ there is a neighborhood $U_{x} \subset U$ of $x$ such that the restricted map $\left.\mathcal{F}\right|_{U_{x}}$ is a Lipschitz map. For a locally Lipschitz map, $\mathcal{F}: U \rightarrow \mathbb{R}^{m}$, defined on the open set $U \subset \mathbb{R}^{n}$ and a compact subset $K \subset U$, the restricted map $\left.\mathcal{F}\right|_{K}$ is 
globally Lipschitz in the sense that $\mathfrak{L}_{\mathcal{F}, K}$, the $K$-Lipschitz constant of the map $\left.\mathcal{F}\right|_{K}$, is given by

$$
\mathfrak{L}_{\mathcal{F}, K}=\sup _{x, y \in K} \frac{|\mathcal{F}(x)-\mathcal{F}(y)|}{|x-y|} .
$$

The vector space of locally Lipschitz mappings from the open set $U \subset \mathbb{R}^{n}$ to $\mathbb{R}^{m}$ is denoted by $\mathfrak{L}\left(U, \mathbb{R}^{m}\right)$. For a compact subset $K \subset U$, define the semi-norm

$$
\|\mathcal{F}\|_{\mathfrak{L}, K}=\max \left\{\left\|\left.\mathcal{F}\right|_{K}\right\|_{\infty}, \mathfrak{L}_{\mathcal{F}, K}\right\},
$$

on $\mathfrak{L}\left(U, \mathbb{R}^{m}\right)$, where,

$$
\left\|\left.\mathcal{F}\right|_{K}\right\|_{\infty}=\sup _{x \in K}|\mathcal{F}(x)| .
$$

The vector space $\mathfrak{L}\left(U, \mathbb{R}^{m}\right)$ is endowed with the strong Lipschitz topology (see [9] for the definition on Riemannian manifolds). It is the analogue of Whitney's topology (strong topology) for the space of differentiable mappings between open sets (see [20, p. 35]) and is defined as follows.

Definition 3.1. Given $\mathcal{F} \in \mathfrak{L}\left(U, \mathbb{R}^{m}\right)$, for some index set $\Lambda$, let $\mathcal{O}=$ $\left\{O_{\lambda}\right\}_{\lambda \in \Lambda}$ be an open, locally finite cover of $U \subset \mathbb{R}^{n}$, and $\mathcal{K}=\left\{K_{\lambda}\right\}_{\lambda \in \Lambda}$ a family of compact subsets covering $U$ such that $K_{\lambda} \subset O_{\lambda}$ and $\delta=\left\{\delta_{\lambda}\right\}_{\lambda \in \Lambda}$ a family of positive numbers. A neighborhood $B^{\mathfrak{L}}(\mathcal{F}, \mathcal{O}, \delta, \mathcal{K})$ of $\mathcal{F}$ in the strong topology is defined as the collection of all $g \in \mathfrak{L}\left(U, \mathbb{R}^{m}\right)$ such that $\|\mathcal{F}-g\|_{\mathfrak{L}, K_{\lambda}}<\delta_{\lambda}$, i.e.

$$
B^{\mathfrak{L}}(\mathcal{F}, \mathcal{O}, \mathcal{K}, \delta)=\left\{g \in \mathfrak{L}\left(U, \mathbb{R}^{n}\right) \mid\|\mathcal{F}-g\|_{\mathfrak{L}, K_{\lambda}}<\delta_{\lambda}, \lambda \in \Lambda\right\} .
$$

For an illustrative description for the strong topology in the case of $C^{0}$ functions, see [20, p. 59].

The following lemma shows the strong character of convergence in the strong topology. For the proof in the case of differentiable mappings, see [23, p. 27] or [11, p. 43].

Lemma 3.2. Let $\left\{\mathcal{F}_{\alpha}\right\}_{\alpha=1}^{\infty}$ be a sequence in $\mathfrak{L}\left(U, \mathbb{R}^{m}\right)$. Then, the sequence converges to $\mathcal{F} \in \mathfrak{L}\left(U, \mathbb{R}^{m}\right)$ in the strong Lipschitz topology, if and only if there exists a compact subset $K \subset U$ such that $\mathcal{F}_{\alpha}$ equals $\mathcal{F}$ on $U \backslash K$ for all but finitely many $\alpha$ 's and $\left.\mathcal{F}_{\alpha}\right|_{K}$ converges to $\left.\mathcal{F}\right|_{K}$ uniformly.

A map $\varphi: U \longrightarrow V$, for open sets $U \subset \mathbb{R}^{n}, V \subset \mathbb{R}^{m}$ and $m \geq n$, is said to be a bi-Lipschitz map if there are numbers $0<c \leq d<\infty$, such that

$$
c \leq \frac{|\varphi(x)-\varphi(y)|}{|x-y|} \leq d, \quad \text { for all } \quad x, y \in U, x \neq y .
$$

(See [19, p. 78] for further discussion.) Setting $L=\max \left\{\frac{1}{c}, d\right\}$, it follows that

$$
\frac{1}{L} \leq \frac{|\varphi(x)-\varphi(y)|}{|x-y|} \leq L, \quad \text { for all } \quad x, y \in U, x \neq y,
$$


and in such a case $\varphi$ is said to be $L$-bi-Lipschitz. (See [19, p. 78] for further discussion.)

Definition 3.3. The map $\mathcal{F}: U \rightarrow V$, where $U \subset \mathbb{R}^{n}$ and $V \subset \mathbb{R}^{m}$ are open sets such that $m \geq n$, is a Lipschitz immersion if for every $x \in U$ there is a neighborhood $U_{x} \subset U$ of $x$ such that $\left.\mathcal{F}\right|_{U_{x}}$ is a bi-Lipschitz map, i.e., there are $0<c_{x} \leq d_{x}<\infty$, and

$$
c_{x} \leq \frac{|\varphi(y)-\varphi(z)|}{|y-z|} \leq d_{x}, \text { for all } y, z \in U_{x}, y \neq z .
$$

Definition 3.4. A Lipschitz map $\varphi: U \rightarrow V$ is said to be a Lipschitz embedding if it is a Lipschitz immersion and a homeomorphism of $U$ onto $\varphi(U)$.

The following theorems pertaining to the set of Lipschitz immersions and Lipschitz embeddings are given in [9] for the setting of Lipschitz manifolds. Their proofs are analogous to the case of differentiable mappings as in [20, p. 36-38].

Theorem 3.5. The set of Lipschitz immersions is an open subset of $\mathfrak{L}\left(U, \mathbb{R}^{m}\right)$ with respect to the strong Lipschitz topology.

Theorem 3.6. The set $\mathfrak{L}_{\mathrm{Em}}\left(U, \mathbb{R}^{m}\right)$ is open in $\mathfrak{L}\left(U, \mathbb{R}^{m}\right)$ with respect to the strong Lipschitz topology .

In the following, a smooth embedding $\varphi$ will be an element of the set

$$
\operatorname{Emb}\left(U, \mathbb{R}^{m}\right)=C^{\infty}\left(U, \mathbb{R}^{m}\right) \cap \mathfrak{L}_{\mathrm{Em}}\left(U, \mathbb{R}^{m}\right),
$$

that is, a Lipschitz embedding whose components are smooth.

\section{The Image of Currents and Homotopy}

Let $U \subset \mathbb{R}^{n}$ and $V \subset \mathbb{R}^{m}$ be open sets, , and let $f: U \rightarrow V$ be a map of class $C^{\infty}$. We recall that for any $\omega \in \mathfrak{D}^{r}(V)$, the pullback of $\omega$ by $f$, is the $r$-form in $U$ denoted by $f^{\#}(\omega)$ such that

$$
\begin{aligned}
f^{\#}(\omega)(x)\left(v_{1} \wedge \cdots \wedge v_{r}\right) & =\omega(f(x))\left(D f(x) v_{1} \wedge \cdots \wedge D f(x) v_{r}\right), \\
& =\omega(f(x))\left(\bigwedge_{r} D f(x)\left(v_{1} \wedge \cdots \wedge v_{r}\right)\right),
\end{aligned}
$$

for any collection of vectors $v_{1}, \ldots, v_{r} \in \mathbb{R}^{n}$. Note that $f^{\#}(\omega)$ need not be an element of $\mathfrak{D}^{r}(U)$. For example, let $f: U \rightarrow \mathbb{R}^{n}$ be the inclusion then for $\omega \in \mathfrak{D}^{r}\left(\mathbb{R}^{n}\right)$ such that $\operatorname{spt}(\omega) \cap U \neq \varnothing$ and $\operatorname{spt}(\omega) \cap\left(\mathbb{R}^{n} \backslash U\right) \neq \varnothing$ then $f^{\#}(\omega)$ is not compactly supported in $U$ an thus $f^{\#}(\omega) \notin \mathfrak{D}^{r}(U)$.

Let $T \in \mathfrak{D}_{r}(U)$ such that $\left.f\right|_{\operatorname{spt}(T)}$ is a proper map. The pushforward of $T$ by $f$ is denoted by $f_{\#}(T) \in \mathfrak{D}_{r}(V)$ and is defined by

$$
f_{\#}(T)(\omega)=T\left(\gamma \wedge f^{\#}(\omega)\right), \quad \text { for all } \omega \in \mathfrak{D}^{r}(V),
$$


where $\gamma \in \mathfrak{D}^{0}(U)$ is any cutoff function satisfying

$$
\operatorname{spt}(T) \cap f^{-1}(\operatorname{spt}(\omega)) \subset \operatorname{Int}\{x \mid \gamma(x)=1\} .
$$

For $T \in \mathfrak{D}_{r}(U)$ with $\operatorname{spt}(T) \subset K$, where $K$ is a compact subset of $U$, we have the following bounds

$$
\begin{aligned}
M\left(f^{\#}(T)\right) & \leq \sup _{x \in K}\|D f(x)\|^{r} M(T), \\
N\left(f^{\#}(T)\right) & \leq \sup _{x \in K}\left\{\|D f(x)\|^{r-1},\|D f(x)\|^{r}\right\} N(T), \\
F_{f\{K\}}\left(f^{\#}(T)\right) & \leq \sup _{x \in K}\left\{\|D f(x)\|^{r},\|D f(x)\|^{r+1}\right\} F_{K}(T) .
\end{aligned}
$$

Let $U \subset \mathbb{R}^{n}$ be an open set and let $f$ and $g$ be smooth maps of $U$ into $\mathbb{R}^{m}$. For an open set $A$ of $\mathbb{R}$ such that $[a, b] \subset A$, a smooth homotopy between the maps $f$ and $g$ is a map

$$
h: A \times U \rightarrow \mathbb{R}^{m}
$$

such that

$$
h(a, x)=f(x), \text { and } h(b, x)=g(x), \quad \text { for all } x \in U .
$$

Henceforth, the following notation will be used

$$
h_{\tau}(x)=h(\tau, x), \text { for all } x \in U,
$$

and

$$
\dot{h}_{\tau}: U \rightarrow \mathbb{R}^{m}, \quad \dot{h}_{\tau}(x)=D h(\tau, x)(1,0)=\frac{\partial h}{\partial \tau}(\tau, x), \text { for all } x \in U .
$$

For $T \in \mathfrak{D}_{r}(U)$ and a homotopy $h$ between $f$ and $g$, the $h$-deformation chain of $T$ is defined as the current

$$
h_{\#}([a, b] \times T) \in \mathfrak{D}_{r+1}\left(\mathbb{R}^{m}\right) .
$$

Traditionally, the interval $[a, b]$ is taken as the unit interval $[0,1]$. The properties $h_{\#}([a, b] \times T)$ are further investigated in [8, Section 4.1.9] and [10, sec. 2.3]. A fundamental tool is the following formula

$$
g_{\#}(T)-f_{\#}(T)=\partial h_{\#}([a, b] \times T)+h_{\#}([a, b] \times \partial T),
$$

which is referred to as the homotopy formula for currents.

Let $\mathcal{F}: U \rightarrow V$ be a locally Lipschitz map, the image of a general $r$-current under a locally Lipschitz map is generally undefined as for any $\omega \in D^{r}(V)$ the fullback $\mathcal{F}^{\#}(\omega)$ need not be a smooth differential $r$-form in $U$, moreover, the coefficients of $\mathcal{F}^{\#}(\omega)$ are not necessary Borel functions. For a normal current $T \in N_{r}(U)$ one can define, see [10, Sec. 2.3], the pushforward of $T$ by the locally Lipschitz map $\mathcal{F}$ as the following weak limit

$$
\mathcal{F}_{\#}(T)(\omega)=\lim _{\rho \rightarrow 0}\left\{\left(\left(\Phi_{\rho} * \mathcal{F}\right)_{\#} T\right)(\omega)\right\},
$$


where $\left\{\Phi_{\rho} * \mathcal{F}\right\}_{\rho}$ is a sequence of smooth approximations obtained by mollification of $\mathcal{F}$ (see [8, Section 4.1.2]). The strong convergence of the sequence is proven by Equation (4.8), as the sequence $\left\{\left(\Phi_{\rho} * \mathcal{F}\right)_{\#} T\right\}_{\rho}$ is shown to be a Cauchy sequence in the Banach space of flat $r$-chains. (See 8 , Section 4.1.14].)

The operator $\mathcal{F}_{\#}: N_{r, K}(U) \rightarrow N_{r, \mathcal{F}\{K\}}(V)$ is continuous with respect to the flat norm and thus extends (we keep the same notation) to $\mathcal{F}_{\#}$ : $F_{r, K}(U) \rightarrow F_{r, \mathcal{F}\{K\}}(V)$. For $T \in \mathfrak{D}_{r}(U)$ with spt $(T) \subset K$, the bounds presented in Equation (4.3) are replaced with

$$
\begin{aligned}
& M\left(\mathcal{F}_{\#}(T)\right) \leq \sup _{x \in K}\left(\mathfrak{L}_{\mathcal{F}, K}\right)^{r} M(T), \\
& N\left(\mathcal{F}_{\#}(T)\right) \leq \sup _{x \in K}\left\{\left(\mathfrak{L}_{\mathcal{F}, K}\right)^{r-1},\left(\mathfrak{L}_{\mathcal{F}, K}\right)^{r}\right\} N(T), \\
& F_{f\{K\}}\left(\mathcal{F}_{\#}(T)\right) \leq \sup _{x \in K}\left\{\left(\mathfrak{L}_{\mathcal{F}, K}\right)^{r},\left(\mathfrak{L}_{\mathcal{F}, K}\right)^{r+1}\right\} F_{K}(T) .
\end{aligned}
$$

For $T \in N_{r, K}(U)$ the existence of $\mathcal{F}_{\#}(T) \in F_{r, \mathcal{F}\{K\}}(V)$, and the second bound in Equation (4.9), imply that $\mathcal{F}_{\#}(T) \in N_{r, \mathcal{F}\{K\}}(V)$.

Alternatively, one may define the pushforward $\mathcal{F}_{\#}(T)$ by utilizing the duality of flat chains and flat forms and setting

$$
\mathcal{F}_{\#}(T)(\omega)=X_{\mathcal{F} \#(\omega)}(T), \quad \text { for all } \omega \in \mathfrak{D}^{r}(\omega) .
$$

By Rademacher's theorem the derivative a Lipschitz mapping exists for $L^{n}$ almost every $x \in U$. Thus, Equation (4.1) is meaningful for $L^{n}$-almost every $x \in U$ and $\mathcal{F}^{\#}(\omega)$ is a flat $r$-form in $U$. It follows that $X_{\mathcal{F} \#(\omega)}$ is a flat $r$-cochain and the action $X_{\mathcal{F}^{\#}(\omega)}(T)$ is well defined. The homotopy theorem for currents, and in particular, the homotopy formula given in Equation (4.8) discussed above for smooth maps, is therefore extended to maps $h: A \times U \rightarrow$ $\mathbb{R}^{m}$ which are locally Lipschitz maps. We note that a similar definition and Wolfe's representation theorem are applied in [30, Sction X.9], to define the pullback of a flat form by a Lipschitz map.

\section{The Lie DeRivative}

In this section we examine the regularity of the Lie derivative of a differential $r$-form. Cartan's (magic) formula is a key element in the following analysis and as a first step we examine the contraction of a differential form by a smooth vector field.

We first introduce a component representation that will be useful throughout this section. The summation convention will be used unless otherwise stated. Let $v=v^{i} e_{i}, e_{i}=\partial / \partial x^{i}$, and $\omega=\omega_{\lambda} d x^{\lambda}$ with $\lambda \in \Lambda(n, r+1)$. Then,

$$
\omega\left\llcorner v=\left(\omega_{\lambda} d x^{\lambda}\right)\left\llcorner\left(v^{i} e_{i}\right)=v^{i} \omega_{\lambda} d x^{\lambda}\left\llcorner e_{i},\right.\right.\right.
$$


and the exterior derivative of $\omega\llcorner v$ is given by

$$
\begin{aligned}
d(\omega\llcorner v) & =\left(v^{i} \omega_{\lambda}\right)_{, j} d x^{j} \wedge\left(d x^{\lambda}\left\llcorner e_{i}\right),\right. \\
& =\left(v_{, j}^{i} \omega_{\lambda}+v^{i} \omega_{\lambda, j}\right) d x^{j} \wedge\left(d x^{\lambda}\left\llcorner e_{i}\right) .\right.
\end{aligned}
$$

Consider the $M_{K}$-seminorm of $\omega\llcorner v$ and recall that

$$
M_{K}\left(\omega\llcorner v)=\operatorname{ess} \sup _{x \in K}\{\|(\omega\llcorner v)(x) \|\} .\right.
$$

The $r$-form $\omega\llcorner v$ has $C(n, r)$ components each of which is a sum of $(n-r)$ terms each of which is a multiplication of a component of $\omega$ with a component of $v$. Hence,

$$
M_{K}\left(\omega\llcorner v) \leq C(n, r) \sup _{x \in K}\|v(x)\| M_{K}(\omega) .\right.
$$

Remark 5.1. For a smooth vector field, $v: U \rightarrow \mathbb{R}^{n}$, and $\omega \in \mathfrak{D}^{r}(U)$ such that $\operatorname{spt}(\omega) \subset K$, note that $\operatorname{spt}(d(\omega\llcorner v)) \subset K$ and the components of $d(v\lrcorner \omega)$ are functions in $C_{0}^{\infty}(U)$. Thus,

$$
M\left(d(\omega\llcorner v))=\operatorname{ess} \sup _{x \in K}\{\| d(\omega\llcorner v)(x) \|\}<\infty .\right.
$$

However, one cannot find a $C<\infty$ such that

$$
\sup _{x \in K}\left\{\|d(\omega\llcorner v)(x) \|\} \leq C\| v \|_{\mathfrak{L}, K} \sup _{x \in K}\{\|d \omega(x)\|\} .\right.
$$

Definition 5.2. The Lie derivative of the differential form $\omega \in \mathfrak{D}^{r}(U)$ with respect to the vector field $v$ on $U$ is the differential $r$-form in $U$ denoted by $\mathcal{L}_{v} \omega$ and defined by

$$
\varphi_{t}^{\#}\left(\mathcal{L}_{v} \omega\right)=\frac{d}{d t}\left(\varphi_{t}^{\#} \omega\right),
$$

where $\varphi: \mathbb{R} \times U \rightarrow U$ is the flow associated with the vector field $v$ [1, p. 370].

The classical Cauchy-Lipschitz theory asserts the existence of a flow for $v$, a time dependent vector field which is Lipschitz continuous in the spatial variable and uniformly continuous with respect to the time variable [21, Sec. IV.1]. The existence of flows for vector fields with reduced regularity, such as vector fields of bounded variation, is an active field of research and for further discussion see [4 and references cited therein. It is noted that in the rest of this work the existence of a flow for the Lipschitz vector field follows from the assumptions regarding the motion described below.

The Lie derivative of a differential form $\omega$ with respect to the vector field $v$ satisfies the identity

$$
\mathcal{L}_{v} \omega=d(\omega\llcorner v)+d \omega\llcorner v,
$$

which is commonly known as Cartan's (magic) formula. Note that in case $v$ is a smooth vector field on $U$, it follows that $\mathcal{L}_{v} \omega \in \mathfrak{D}^{r}(U)$ for every $\omega \in \mathfrak{D}^{r}(U)$. 
Lemma 5.3. Let $\omega \in \mathfrak{D}^{r}(U)$ and let $v: U \rightarrow \mathbb{R}^{n}$ be a smooth vector field. Then, there exists a constant $C(n, r)$ such that

$$
M_{K}\left(\mathcal{L}_{v} \omega\right) \leq C(n, r) S_{K}(\omega)\|v\|_{\mathfrak{L}, K} .
$$

Moreover, the Lie derivative of a sharp $r$-form with respect to a smooth vector field, taken in the weak sense, is an r-form of locally finite mass.

Proof. By Equations (5.1), (5.2) and (5.6), a local representation of the Lie derivative $\mathcal{L}_{v} \omega$ is given by

$$
\mathcal{L}_{v} \omega=D(\omega)(v)+v_{, j}^{i} \omega_{\lambda} d x^{j} \wedge\left(d x^{\lambda}\left\llcorner e_{i}\right) .\right.
$$

For a given $\lambda \in \bigwedge(n, r)$, the contraction $d x^{\lambda}\left\llcorner e_{i}\right.$ does not vanish for $r$ base vectors $e_{k}$.For a selection of $\lambda$ and $k$ such that $d x^{\lambda}\left\llcorner e_{k} \neq 0\right.$, the wedge product $d x^{j} \wedge\left(d x^{\lambda}\left\llcorner e_{k}\right)\right.$ will not vanish for a subset of $\left\{d x^{j}\right\}$ containing $(n-(r-1))$ elements. Hence,

$$
\begin{aligned}
M_{K}\left(\mathcal{L}_{v} \omega\right) & \leq M_{K}(D(\omega)(v))+M_{K}\left(v_{, j}^{i} \omega_{\lambda} d x^{j} \wedge\left(d x^{\lambda}\left\llcorner e_{i}\right)\right),\right. \\
& \leq \mathfrak{L}_{\omega, K}\|v\|_{\infty, K}+\frac{n !}{r !(n-r) !} r(n-(r-1)) M_{K}(\omega) \mathfrak{L}_{v, K}, \\
& \leq C(n, r)\|v\|_{\mathfrak{L}, K} S_{K}(\omega) .
\end{aligned}
$$

The extension to sharp forms follows from Rademacher's theorem which implies that for a sharp form $\omega, D \omega$ exists almost everywhere in $U$.

For a smooth vector field $v: U \rightarrow \mathbb{R}^{n}$ defined on the open set $U \subset \mathbb{R}^{n}$ and an $r$-current $T \in \mathfrak{D}_{r}(U)$, the $(r+1)$-current $v \wedge T$ is defined by

$$
v \wedge T(\omega)=T\left(\omega\llcorner v), \quad \text { for all } \omega \in \mathfrak{D}^{r+1}(U) .\right.
$$

As $v$ is a smooth vector field it follows that $\omega\left\llcorner v \in \mathfrak{D}^{r}(U)\right.$ and $T(\omega\llcorner v)$ is well defined.

By the remark preceding Definition 5.2, it follows that given $T \in F_{r, K}(U)$ and a smooth vector field $v: U \rightarrow \mathbb{R}^{n}$, the $(r+1)$-current $v \wedge T$ is not necessarily a flat $(r+1)$-chain. Moreover, even if $T \in N_{r, K}(U)$ the current $v \wedge T$ may not be a flat $(r+1)$-chain. The analysis of $v \wedge T$ is a key element in what follows and the foregoing remark is an example for the restricted applicability of the flat norm.

The contraction of a vector field and a differential form defined above may be extended to include locally Lipschitz vector fields. Let $\omega \in \mathfrak{D}^{r+1}(U)$, where $U \subset \mathbb{R}^{n}$ is an open set, and let $v: U \rightarrow \mathbb{R}^{n}$ be a locally Lipschitz vector field. Define $\omega\llcorner v$ as the pointwise limit of the contractions with the mollified vector fields $\Phi_{\rho} * v$, i.e.,

$$
\omega\left\llcorner v(x)=\lim _{\rho \rightarrow 0}\left\{\omega(x)\left\llcorner\left(\Phi_{\rho} * v\right)(x)\right\} .\right.\right.
$$


We note that the convergence of the above limit is locally uniform with respect to $x$. In a similar manner to the estimate in Equation (5.4) we have

$$
\begin{aligned}
M_{K}(\omega\llcorner v) & =\lim _{\rho \rightarrow 0} M_{K}\left(\omega\left\llcorner\left(\Phi_{\rho} * v\right)\right)\right. \\
& \leq \lim _{\rho \rightarrow 0} C(n, r) \operatorname{ess} \sup _{x \in K}\left\|\Phi_{\rho} * v(x)\right\| M_{K}(\omega) \\
& =C(n, r)\|v\|_{\infty, K} M_{K}(\omega) .
\end{aligned}
$$

For the Lie derivative of $\omega \in \mathfrak{D}^{r}(U)$ with respect to the Lipschitz vector field we have

$$
\mathcal{L}_{v} \omega=\lim _{\rho \rightarrow 0}\left(\mathcal{L}_{\Phi_{\rho} * v} \omega\right)=\lim _{\rho \rightarrow 0}\left(d \left(\omega\left\llcorner\left(\Phi_{\rho} * v\right)\right)+d \omega\left\llcorner\left(\Phi_{\rho} * v\right)\right),\right.\right.
$$

where the above limit is taken with respect to the $M_{K}(\cdot)$-semi-norm. The existence of the limit follows from the fact that

$$
\lim _{\rho \rightarrow 0}\left\|\Phi_{\rho} * v-v\right\|_{\mathfrak{L}, K}=0,
$$

and the bound given in Equation (5.9). Moreover, the bound in Equation (5.9) holds for locally Lipschitz vector field.

\section{Smooth Configurations and Motions}

Let $\mathcal{B} \subset \mathbb{R}^{n}$ and $\mathcal{I} \subset \mathbb{R}$ bounded open subsets. Recalling (3.9), a smooth motion $m$ defined over the time interval $\mathcal{I}$ is viewed as a curve

$$
m: \mathcal{I} \rightarrow \operatorname{Emb}\left(\mathcal{B}, \mathbb{R}^{n}\right) .
$$

We assume that the motion $m$ is a $C^{1}$-curve with respect to strong Lipschitz topology, that is, the derivative of the curve, denoted by $\dot{m}$, is viewed as a curve

$$
\dot{m}: \mathcal{I} \rightarrow C^{\infty}\left(\mathcal{B}, \mathbb{R}^{n}\right) \cap \mathfrak{L}\left(\mathcal{B}, \mathbb{R}^{n}\right),
$$

which is continuous with respect to the strong Lipschitz topology.

The motion $m$, induces a map

$$
\varphi: \mathcal{I} \times \mathcal{B} \rightarrow \mathbb{R}^{n}
$$

by

$$
\varphi(\tau, x)=m(\tau)(x), \quad \text { for all } \tau \in \mathcal{I}, x \in \mathcal{B},
$$

and so

$$
\frac{\partial \varphi}{\partial t}(\tau, x)=\dot{m}(\tau)(x), \quad \text { for all } \tau \in \mathcal{I}, x \in \mathcal{B} .
$$

It follows from Lemma 3.2 , that there exists a compact subset $K_{m} \subset \mathcal{B}$ such that for any $x \notin K_{m}$ and every $t, t^{\prime} \in \mathcal{I}$

$$
\varphi(t, x)=\varphi\left(t^{\prime}, x\right) .
$$

Hence, for $x \notin K_{m}$ and $t \in \mathcal{I}$

$$
\dot{\varphi}(t, x)=0 .
$$

For some $t \in \mathcal{I}$, set $\mathcal{B}^{\prime}=\varphi_{t}\{\mathcal{B}\}$ and $K_{m}^{\prime}=\varphi_{t}\left\{K_{m}\right\}$. By the preceding argument, $B^{\prime}$ and $K_{m}^{\prime}$ are independent of the particular choice of $t \in \mathcal{I}$. 
Remark 6.1. Equations (6.3, 6.4) and the existence of $K_{m}$ are key features of the motion examined and stem from the use of the strong Lipschitz topology. A drawback to the use of the strong Lipschitz topology is in the relatively small supply of converging sequences of maps in the form of Equation (6.2) converging to a motion as given in Equation (6.1).

For each $t \in \mathcal{I}, \varphi_{t}=m(t) \in \operatorname{Emb}\left(\mathcal{B}, \mathbb{R}^{n}\right)$, so there exists an inverse $\eta_{t}$ : image $\left(\varphi_{t}\right)=\mathcal{B}^{\prime} \rightarrow \mathcal{B}$ such that $\varphi_{t} \circ \eta_{t}=I_{\mathcal{B}^{\prime}}$ with $I_{\mathcal{B}^{\prime}}$ the identity map on the set $\mathcal{B}^{\prime}$. Consider the vector field

$$
v_{t}: \mathcal{B}^{\prime} \rightarrow \mathbb{R}^{n}, \quad v_{t}=\dot{\varphi}_{t} \circ \eta_{t},
$$

viewed as a vector field on $\mathcal{B}^{\prime}$ such that $v_{t}(y)=0$ for every $x \in \mathcal{B}^{\prime} \backslash K_{m}^{\prime}$. The vector field $v_{t}$ is naturally extended to a vector field $\hat{v}_{t}: \mathbb{R}^{n} \rightarrow \mathbb{R}^{n}$, by setting

$$
\hat{v}_{t}(x)= \begin{cases}v_{t}(x), & x \in \mathcal{B}^{\prime}, \\ 0, & x \notin \mathcal{B}^{\prime} .\end{cases}
$$

Thus, $\hat{v}_{t}$ is a smooth vector field which vanishes on $\mathbb{R}^{n} \backslash K_{m}^{\prime}$. It follows that,

$$
\hat{v}: \mathcal{I} \times \mathbb{R}^{n} \rightarrow \mathbb{R}^{n}
$$

is a time dependent Lipschitz vector field defined on $\mathbb{R}^{n}$.

For $s, t \in \mathcal{I}$, define

$$
J_{s, t}(x)= \begin{cases}\varphi_{s} \circ \eta_{t}(x), & x \in \mathcal{B}^{\prime}, \\ x, & x \notin \mathcal{B}^{\prime}\end{cases}
$$

As shown in [7],

$$
\frac{\partial J_{s, t}}{\partial s}(x)=\hat{v}_{s}\left(J_{s, t}(x)\right), \quad J_{t, t}(x)=x .
$$

The map $J_{s, t}$, is the flow associated with the time dependent vector field $\hat{v}$. For $\omega \in \mathfrak{D}^{r}\left(\mathbb{R}^{n}\right)$,

$$
\left.\frac{\partial\left(J_{\tau, t}^{\#} \omega\right)}{\partial \tau}\right|_{\tau=s}=J_{s, t}^{\#}\left(\mathcal{L}_{\hat{v}_{s}} \omega\right),
$$

and as $\varphi_{\tau}^{\#} \omega=\varphi_{t}^{\#}\left(J_{\tau, t}^{\#} \omega\right)$, it follows from Definition 5.5 and a direct computation that

$$
\left.\frac{\partial\left(\varphi_{\tau}^{\#} \omega\right)}{\partial \tau}\right|_{\tau=t}=\varphi_{t}^{\#}\left(\mathcal{L}_{\hat{v}_{t}} \omega\right) .
$$

We now derive a representation formula for the $\varphi$-deformation chain associated with the motion $\varphi: \mathcal{I} \times \mathcal{B} \rightarrow \mathbb{R}$ and a general current $T \in \mathfrak{D}_{r}(\mathcal{B})$. 
Let $[a, b] \subset \mathcal{I}$, then, applying Equation (2.25) to $\omega \in \mathfrak{D}^{r+1}\left(\mathbb{R}^{n}\right)$, one has

$$
\begin{aligned}
\varphi_{\#}([a, b] \times T)(\omega) & =([a, b] \times T)\left(\varphi^{\#}(\omega)\right), \\
& =\int_{a}^{b} T\left(\varphi_{\tau}^{\#}(\omega)_{H}\right) d L_{\tau}^{1}, \\
& =\int_{a}^{b} T\left(\varphi_{\tau}^{\#}(\omega)\left\llcorner e_{t}\right) d L_{\tau}^{1} .\right.
\end{aligned}
$$

In order to examine the $r$-form $\varphi_{\tau}^{\#}(\omega)\left\llcorner e_{t}\right.$, we apply it to an $r$-vector $\xi$ which we can assume to be "space-like", that is, $\xi=v_{1} \wedge \cdots \wedge v_{r}$ with $v_{i} \in \mathbb{R}^{n}$, for $i=1, \ldots, r$. Otherwise, $\left(\varphi_{\tau}^{\#}(\omega)\left\llcorner e_{t}\right)(\xi)=0\right.$, identically. One obtains,

$$
\begin{aligned}
\left(\varphi_{\tau}^{\#}(\omega)(x)\left\llcorner e_{t}\right)(\xi)\right. & =\varphi^{\sharp}(\omega)(\tau, x)\left(e_{t} \wedge v_{1} \wedge \cdots \wedge v_{r}\right), \\
& =\omega \circ \varphi_{\tau}(x)\left(D \varphi(\tau, x)\left(e_{t}\right) \wedge \bigwedge_{r} D \varphi_{\tau}(x)(\xi)\right), \\
& =\omega \circ \varphi_{\tau}(x)\left(\dot{\varphi}_{\tau}(x) \wedge \bigwedge_{r} D \varphi_{\tau}(x)(\xi)\right), \\
& =\left(\left(\omega \circ \varphi_{\tau}\right)\left\llcorner\dot{\varphi}_{\tau}\right)(x)\left(\bigwedge_{r} D \varphi_{\tau}(x)(\xi)\right) .\right.
\end{aligned}
$$

As $\dot{\varphi}_{\tau}(x)=v_{\tau}\left(\varphi_{\tau}(x)\right)=\hat{v}_{\tau} \circ \varphi_{\tau}(x)$,

$$
\begin{aligned}
\left(\varphi^{\#}(\omega)(\tau, x)\left\llcorner e_{t}\right)(\xi)\right. & =\left(\left(\omega \circ \varphi_{\tau}\right)\left\llcorner\left(\hat{v}_{\tau} \circ \varphi_{\tau}\right)\right)(x)\left(\bigwedge_{r} D \varphi_{\tau}(x)(\xi)\right),\right. \\
& =\left(\omega\left\llcorner\hat{v}_{\tau}\right) \circ \varphi_{\tau}(x)\left(\bigwedge_{r} D \varphi_{\tau}(x)(\xi)\right),\right. \\
& =\varphi_{\tau}^{\#}\left(\omega\left\llcorner\hat{v}_{\tau}\right)(x)(\xi) .\right.
\end{aligned}
$$

It is concluded that,

$$
\varphi_{\tau}^{\#}(\omega)\left\llcorner e_{t}=\varphi_{\tau}^{\#}\left(\omega\left\llcorner\hat{v}_{\tau}\right) .\right.\right.
$$

Returning to Equation (6.10), note that the integrand may be rewritten as

$$
\begin{aligned}
T\left(\varphi_{\tau}^{\#}(\omega)\left\llcorner e_{t}\right)\right. & =T\left(\varphi_{\tau}^{\#}\left(\omega\left\llcorner\hat{v}_{\tau}\right)\right),\right. \\
& =\varphi_{\tau \#}(T)\left(\omega\left\llcorner\hat{v}_{\tau}\right),\right. \\
& =\hat{v}_{\tau} \wedge \varphi_{\tau \#}(T)(\omega),
\end{aligned}
$$

and Equation (6.10) assumes the form

$$
\varphi_{\#}([a, b] \times T)(\omega)=\int_{a}^{b}\left(\hat{v}_{\tau} \wedge \varphi_{\tau \#} T\right)(\omega) d L_{\tau}^{1} .
$$


Applying (4.8) to (4.8), one finally has,

$$
\begin{aligned}
\left(\varphi_{b \#}(T)-\varphi_{a \#}(T)\right) \omega & =\left(\partial \varphi_{\#}([a, b] \times T)+\varphi_{\#}([a, b] \times \partial T)\right) \omega, \\
& =\int_{a}^{b}\left[\left(\hat{v}_{\tau} \wedge \varphi_{\tau \#} T\right)(d \omega)+\left(\hat{v}_{\tau} \wedge \varphi_{\tau \#} \partial T\right)(\omega)\right] d L_{\tau}^{1}, \\
& =\int_{a}^{b}\left[( \varphi _ { \tau \# } T ) \left(d \omega\left\llcorner\hat{v}_{\tau}+d\left(\omega\left\llcorner\hat{v}_{\tau}\right)\right)\right] d L_{\tau}^{1},\right.\right. \\
& =\int_{a}^{b}\left[\left(\varphi_{\tau \#} T\right)\left(\mathcal{L}_{\hat{v}_{\tau}} \omega\right)\right] d L_{\tau}^{1} .
\end{aligned}
$$

\section{The Kinematics of Currents under a Smooth Motion}

This section is devoted to the examination of kinematic properties of generalized domains. A generalized $r$-dimensional oriented domain, is naturally viewed as an $r$-current. In the selection of the appropriate class of domains, the collection of all $r$-currents is far greater than what we would consider as suitable. The selection of the appropriate class is motivated by the following guidelines. Firstly, a current representing a generalized domain must have a local character, at least in some measure theoretic sense. Secondly, such a current must have a definite, quantitative notion of a boundary.Finally, such a current should be well behaved under the image of a Lipschitz map 1 The introductory discussion in Sections 2 and 4 indicates that a convenient choice for the class of domains is the collection of flat chains of finite mass. Thus, a prototypical control volume $T$, is viewed as a flat $r$-chain of finite mass in $\mathcal{B}$. Using the properties of a motion we outlined above and the corresponding notation of Section 6 , we consider $T \in F_{r, K_{m}}(\mathcal{B})$, where $K_{m} \subset \mathcal{B}$ is the compact set containing the region where the motion is nontrivial.

Remark 7.1. Let $T \in F_{r}(\mathcal{B})$ and $\gamma: \mathcal{B} \rightarrow \mathbb{R}$ a locally Lipschitz function, the multiplication $\gamma \wedge T$ is flat $r$-chain. Thus, a flat $r$-chain may represent not only a geometric domain but may also be represent some intensive property. See [6] for further details.

Consider a map $\varphi$ induced by a motion as defined in Equation (6.2) and a flat chain $T \in F_{r, K_{m}}(\mathcal{B})$ such that $M(T)<\infty$. The curve $t \mapsto \varphi_{t \#}(T)$ will be viewed in this work as the time evolution of the control volume represented by the current $T$.

Lemma 7.2. Let $\varphi$ be the map associated with a motion as defined by Equation (6.2) and $T \in F_{r, K_{m}}(\mathcal{B})$ a flat chain of finite mass. The curve induced by the pushforward $t \mapsto \varphi_{t \#} T$ is a continuous curve with respect to the $M_{K_{m}^{\prime}}$ norm on $\mathfrak{D}_{r}\left(\mathbb{R}^{n}\right)$.

\footnotetext{
${ }^{1}$ We feel that these requirement are in the spirit put forth by Noll \& Virga in [24] where the class admissible bodies should include all those that can be imagined by an engineer but exclude those that can be dreamt up only by an ingenious mathematician.
} 
Proof. Let $t \in \mathcal{I}$ and select $\varepsilon$ such that $t+\varepsilon \in \mathcal{I}$. Then,

$$
\begin{aligned}
M_{K_{m}^{\prime}}\left(\varphi_{t+\varepsilon \#} T-\varphi_{t \#} T\right) & =\sup _{\omega \in \mathfrak{D}^{r}\left(\mathbb{R}^{n}\right)} \frac{\left(\varphi_{t+\varepsilon \#} T-\varphi_{t \#} T\right) \omega}{M_{K_{m}^{\prime}}(\omega)}, \\
& =\sup _{\omega \in \mathfrak{D}^{r}\left(\mathbb{R}^{n}\right)} \frac{T\left(\varphi_{t+\varepsilon}^{\#} \omega-\varphi_{t}^{\#} \omega\right)}{M_{K_{m}^{\prime}}(\omega)}, \\
& \leq \sup _{\omega \in \mathfrak{D}^{r}\left(\mathbb{R}^{n}\right)} \frac{M(T) M_{K_{m}}\left(\varphi_{t+\varepsilon}^{\#} \omega-\varphi_{t}^{\#} \omega\right)}{M_{K_{m}^{\prime}}(\omega)},
\end{aligned}
$$

where the last line follows from the integral representation of $T$. For the term $M_{K_{m}}\left(\varphi_{t+\varepsilon}^{\#} \omega-\varphi_{t}^{\#} \omega\right)$, a direct computation shows that

$$
\begin{aligned}
& M_{K_{m}}\left(\varphi_{t+\varepsilon}^{\#} \omega-\varphi_{t}^{\#} \omega\right) \\
& =\sup _{x \in K_{m}}\left\{\sup _{\xi}\left\{\omega\left(\varphi_{t+\varepsilon}(x)\right)\left[\bigwedge_{r} D \varphi_{t+\varepsilon}(x)(\xi)\right]-\omega\left(\varphi_{t}(x)\right)\left[\bigwedge_{r} D \varphi_{t}(x)(\xi)\right]\right\}\right\}, \\
& \leq \sup _{x \in K_{m}}\left\{\sup _{\xi}\left\{\omega\left(\varphi_{t+\varepsilon}(x)\right)\left[\bigwedge_{r} D \varphi_{t+\varepsilon}(x)(\xi)\right]-\omega\left(\varphi_{t+\varepsilon}(x)\right)\left[\bigwedge_{r} D \varphi_{t}(x)(\xi)\right]\right\}\right\} \\
& +\sup _{x \in K_{m}}\left\{\sup _{\xi}\left\{\omega\left(\varphi_{t+\varepsilon}(x)\right)\left[\bigwedge_{r} D \varphi_{t}(x)(\xi)\right]-\omega\left(\varphi_{t}(x)\right)\left[\bigwedge_{r} D \varphi_{t}(x)(\xi)\right]\right\}\right\}, \\
& \leq \sup _{x \in K_{m}}\left\{\sup _{\xi}\left\{\omega\left(\varphi_{t+\varepsilon}(x)\right)\left[\bigwedge_{r} D\left(\varphi_{t+\varepsilon}-\varphi_{t}\right)(x)(\xi)\right]\right\}\right\} \\
& \left.\left.\quad+\sup _{x \in K_{m}}\left\{\sup _{\xi}\left\{\left(\omega\left(\varphi_{t+\varepsilon}(x)\right)-\omega\left(\varphi_{t}(x)\right)\right)\right] \bigwedge_{r} D \varphi_{t}(x)(\xi)\right]\right\}\right\}, \\
& \leq M_{K_{m}^{\prime}}(\omega)\left(\mathfrak{L}_{\varphi_{t+\varepsilon}-\varphi_{t}, K_{m}}\right)^{r}+\left(\mathfrak{L}_{\varphi_{t}, K_{m}}\right)^{r} M_{K_{m}^{\prime}}\left(\omega \circ \varphi_{t+\varepsilon}-\omega \circ \varphi_{t}\right) .
\end{aligned}
$$

By the continuity of the motion with respect to the strong Lipschitz topology it follows that as $\varepsilon \rightarrow 0$ we have $\left(\mathfrak{L}_{\varphi_{t+\varepsilon}-\varphi_{t}, K_{m}}\right)^{r} \rightarrow 0$. For the second term, as $\left\|\varphi_{t+\varepsilon}-\varphi_{t}\right\|_{\infty, K_{m}} \rightarrow 0$, and since $\omega$ is smooth, it follows that $M_{K_{m}^{\prime}}\left(\omega \circ \varphi_{t+\varepsilon}-\omega \circ \varphi_{t}\right) \rightarrow 0$, which completes the proof.

As considered in [7, for all $\omega \in \mathfrak{D}^{r}(U)$,

$$
\begin{aligned}
\left.\frac{d}{d t}\left(\left(\varphi_{t \#} T\right)(\omega)\right)\right|_{t=\tau} & =\left.\frac{d}{d t}\left(T \varphi_{t}^{\#}(\omega)\right)\right|_{t=\tau}, \\
& =T\left(\left.\frac{d}{d t} \varphi_{t}^{\#}(\omega)\right|_{t=\tau}\right), \\
& =T\left(\varphi_{\tau}^{\#}\left(\mathcal{L}_{\hat{v}_{\tau}} \omega\right)\right) .
\end{aligned}
$$


Thus, using Equation (5.6)

$$
\begin{aligned}
T\left(\varphi_{\tau}^{\#}\left(\mathcal{L}_{\hat{v}_{\tau}} \omega\right)\right) & =\varphi_{\tau \#} T\left(d \left(\omega\left\llcorner\hat{v}_{\tau}\right)+(d \omega)\left\llcorner\hat{v}_{\tau}\right),\right.\right. \\
& =\left(\hat{v}_{\tau} \wedge \partial\left(\varphi_{\tau \#} T\right)+\partial\left(\hat{v}_{\tau} \wedge \varphi_{\tau \#} T\right)\right) \omega .
\end{aligned}
$$

The foregoing result applies to general currents and is not restricted to flat chains of finite mass. It may also be written with the introduction of $\mathcal{R}_{\hat{v}_{t}}=$ $\left(\mathcal{L}_{\hat{v}_{t}}\right)^{*}$ as the dual operator of the Lie derivative, that is

$$
T\left(\varphi_{t}^{\#}\left(\mathcal{L}_{\hat{v}_{t}} \omega\right)\right)=\mathcal{R}_{\hat{v}_{t}}\left(\varphi_{t \#} T\right) \omega .
$$

Thus, for any de Rham current $T \in \mathfrak{D}_{r}\left(\mathbb{R}^{n}\right)$

$$
\mathcal{R}_{\hat{v}_{t}}(T)=\hat{v}_{t} \wedge \partial T+\partial\left(\hat{v}_{t} \wedge T\right) .
$$

It is observed that similar results have been reported in [5].

The previous analysis shows that the derivative $d\left(\varphi_{t \#} T\right) / d t$ converges in the topology of $\mathfrak{D}_{r}\left(\mathbb{R}^{n}\right)$. The following theorem considers the convergence of the limit above in the sharp norm topology.

Theorem 7.3. Let $\varphi$ be a map associated with a motion as defined by Equation (6.2) and let $T \in F_{r, K_{m}}(\mathcal{B})$ be a flat chain of finite mass. The derivative $\left.\frac{d}{d t}\left(\varphi_{t \#} T\right)\right|_{t=\tau}$, exists in the topology of $S_{K_{m}^{\prime}}\left(\mathbb{R}^{n}\right)$ and is given by

$$
\left.\frac{d}{d t}\left(\varphi_{t \#} T\right)\right|_{t=\tau}=\partial\left(\hat{v}_{\tau} \wedge \varphi_{\tau \#} T\right)+\hat{v}_{\tau} \wedge \partial\left(\varphi_{\tau \#} T\right) .
$$

Proof. By the homotopy formula (4.8), we may write

$$
\frac{d}{d t}\left(\varphi_{t \#} T\right)=\lim _{\varepsilon \rightarrow 0} \frac{\partial \varphi_{\#}([\tau, \tau+\varepsilon] \times T)+\varphi_{\#}([\tau, \tau+\varepsilon] \times \partial T)}{\varepsilon} .
$$

Thus,

$$
\begin{aligned}
& \frac{1}{\varepsilon} S_{K_{m}^{\prime}}\left(\varphi_{t+\varepsilon \#} T-\varphi_{t \#} T-\varepsilon\left(\partial\left(\hat{v}_{t} \wedge \varphi_{t \#} T\right)+\hat{v}_{t} \wedge \partial\left(\varphi_{t \#} T\right)\right)\right) \\
& =\frac{1}{\varepsilon} \sup _{\omega \in \mathfrak{D}^{r}\left(\mathbb{R}^{n}\right)} \frac{\left(\varphi_{t+\varepsilon \#} T-\varphi_{t \#} T-\varepsilon\left(\partial\left(\hat{v}_{t} \wedge \varphi_{t \#} T\right)+\hat{v}_{t} \wedge \partial\left(\varphi_{t \#} T\right)\right)\right) \omega}{S_{K_{m}^{\prime}}(\omega)}, \\
& =\frac{1}{\varepsilon} \sup _{\omega \in \mathfrak{D}^{r}\left(\mathbb{R}^{n}\right)}\left\{\frac{\int_{t}^{t+\varepsilon}\left[\left(\hat{v}_{\tau} \wedge \varphi_{\tau \#} T\right)(d \omega)+\left(\hat{v}_{\tau} \wedge \varphi_{\tau \#} \partial T\right)(\omega)\right] d L_{\tau}^{1}}{S_{K_{m}^{\prime}}(\omega)}\right. \\
& \left.-\frac{\varepsilon\left(\hat{v}_{t} \wedge \varphi_{t \#} T\right)(d \omega)+\hat{v}_{t} \wedge \partial\left(\varphi_{t \#} T\right) \omega}{S_{K_{m}^{\prime}}(\omega)}\right\}, \\
& \leq \frac{1}{\varepsilon} \sup _{\omega \in \mathfrak{D}^{r}\left(\mathbb{R}^{n}\right)}\left\{\frac{\int_{t}^{t+\varepsilon}\left[T\left(\varphi_{\tau}^{\#} \mathcal{L}_{\hat{v}_{\tau}} \omega\right)\right] d L_{\tau}^{1}-\varepsilon T\left(\left(\varphi_{t}^{\#} \mathcal{L}_{\hat{v}_{t}} \omega\right)\right)}{S_{K_{m}^{\prime}}(\omega)}\right\}, \\
& \leq \sup _{\omega \in \mathfrak{D}^{r}\left(\mathbb{R}^{n}\right)}\left\{M(T) \sup _{s \in[t, t+\varepsilon]} \frac{M_{K_{m}}\left(\varphi_{s}^{\#} \mathcal{L}_{\hat{v}_{s}} \omega-\varphi_{t}^{\#} \mathcal{L}_{\hat{v}_{t}} \omega\right)}{S_{K_{m}^{\prime}}(\omega)}\right\} .
\end{aligned}
$$


For the term $M_{K_{m}}\left(\varphi_{s}^{\#} \mathcal{L}_{\hat{v}_{s}} \omega-\varphi_{t}^{\#} \mathcal{L}_{\hat{v}_{t}} \omega\right)$, one has the estimate

$$
\begin{aligned}
M_{K_{m}}\left(\varphi_{s}^{\#} \mathcal{L}_{\hat{v}_{s}} \omega-\varphi_{t}^{\#} \mathcal{L}_{\hat{v}_{t}} \omega\right) \\
\quad \leq M_{K_{m}}\left(\varphi_{s}^{\#} \mathcal{L}_{\hat{v}_{s}} \omega-\varphi_{s}^{\#} \mathcal{L}_{\hat{v}_{t}} \omega+\varphi_{s}^{\#} \mathcal{L}_{\hat{v}_{t}} \omega-\varphi_{t}^{\#} \mathcal{L}_{\hat{v}_{t}} \omega\right) \\
\leq M_{K_{m}}\left(\varphi_{s}^{\#} \mathcal{L}_{\hat{v}_{s}} \omega-\varphi_{s}^{\#} \mathcal{L}_{\hat{v}_{t}} \omega\right)+M_{K_{m}}\left(\varphi_{s}^{\#} \mathcal{L}_{\hat{v}_{t}} \omega-\varphi_{t}^{\#} \mathcal{L}_{\hat{v}_{t}} \omega\right) \\
\leq M_{K_{m}}\left(\varphi_{s}^{\#}\left(\mathcal{L}_{\hat{v}_{s}-\hat{v}_{t}} \omega\right)\right)+M_{K_{m}}\left(\varphi_{s}^{\#} \mathcal{L}_{\hat{v}_{t}} \omega-\varphi_{t}^{\#} \mathcal{L}_{\hat{v}_{t}} \omega\right) .
\end{aligned}
$$

By Equation (5.7),

$$
\begin{aligned}
M_{K_{m}}\left(\varphi_{s}^{\#}\left(\mathcal{L}_{\hat{v}_{s}-\hat{v}_{t}} \omega\right)\right) & \leq\left(\mathfrak{L}_{\varphi_{s}, K_{m}}\right)^{r} M_{K_{m}^{\prime}}\left(\mathcal{L}_{\hat{v}_{s}-\hat{v}_{t}} \omega\right), \\
& \leq\left(\mathfrak{L}_{\varphi_{s}, K_{m}}\right)^{r} C(n, r) S_{K_{m}^{\prime}}(\omega)\left\|\hat{v}_{s}-\hat{v}_{t}\right\|_{\mathfrak{L}, K_{m}^{\prime}},
\end{aligned}
$$

and since $\left\|\hat{v}_{s}-\hat{v}_{t}\right\|_{\mathfrak{L}, K_{m}^{\prime}} \rightarrow 0$ as $s \rightarrow t$, it follows that

$$
\lim _{s \rightarrow t} M_{K_{m}}\left(\varphi_{s}^{\#}\left(\mathcal{L}_{\hat{v}_{s}-\hat{v}_{t}} \omega\right)\right)=0
$$

Applying Lemma 7.2, it follows that

$$
\lim _{s \rightarrow t} M_{K_{m}}\left(\varphi_{s}^{\#} \mathcal{L}_{\hat{v}_{t}} \omega-\varphi_{t}^{\#} \mathcal{L}_{\hat{v}_{t}} \omega\right)=0
$$

Setting

$$
T_{k}=\frac{\partial \varphi_{\#}\left(\left[t, t+\frac{1}{k}\right] \times T\right)+\varphi_{\#}\left(\left[t, t+\frac{1}{k}\right] \times \partial T\right)}{\frac{1}{k}},
$$

we obtain a sequence $\left\{T_{k}\right\}$ of flat $r$-chains converging to $\hat{v}_{t} \wedge \partial\left(\varphi_{t \#} T\right)+$ $\partial\left(\hat{v}_{t} \wedge \varphi_{t \#} T\right)$ in $S_{r}\left(\mathbb{R}^{n}\right)$. As normal currents are dense in the space of flat chains one may obtain a sequence of normal $r$-currents converging to $\hat{v}_{t} \wedge$ $\partial\left(\varphi_{t \#} T\right)+\partial\left(\hat{v}_{t} \wedge \varphi_{t \#} T\right)$ in the $S_{K_{m}^{\prime}}$-norm.

Remark 7.4. Note that the map $t \mapsto \partial\left(\hat{v}_{t} \wedge \varphi_{t \#} T\right)+\hat{v}_{t} \wedge \partial\left(\varphi_{t \#} T\right)$ need not be continuous with respect to the $S_{K_{m}^{\prime}}$-norm, as $S_{K^{\prime}}(\omega)$ may depend of the second derivative of $\omega \in \mathfrak{D}^{r}\left(\mathbb{R}^{n}\right)$. As an example, consider the simple case of $\mathcal{B} \subset \mathbb{R}$, and $T \in N(U)$ given by $T(\omega)=\omega\left(x_{0}\right)+d \omega\left(x_{0}\right)$. For $v=e_{1}$,

$$
T\left(\mathcal{L}_{v} \omega\right)=D \omega\left(x_{0}\right)+D^{2} \omega\left(x_{0}\right) .
$$

\section{Lipschitz Type Configurations and Motion}

This section extends the foregoing discussion to non smooth motions. In particular, configurations represented by bi-Lipschitz maps, as well as the corresponding motions, will be examined.

The definition of a motion, as introduced in Section 6, is generalized by considering curves of the form

$$
m: \mathcal{I} \rightarrow \mathfrak{L}_{\mathrm{Em}}\left(\mathcal{B}, \mathbb{R}^{n}\right),
$$


which we assume are continuously differentiable with respect to the strong Lipschitz topology. Thus, the time derivative of the map is

$$
\dot{m}: \mathcal{I} \rightarrow \mathfrak{L}(\mathcal{B}, \mathbb{R}),
$$

a continuous curve with respect to the strong Lipschitz topology. The motion $m$ induces a map

$$
\kappa: \mathcal{I} \times \mathcal{B} \rightarrow \mathbb{R}^{n}
$$

such that

$$
\kappa(\tau, x)=m(\tau)(x), \quad \text { for all } \tau \in \mathcal{I}, x \in \mathcal{B},
$$

and so

$$
\dot{\kappa}(\tau, x)=\dot{m}(\tau)(x), \quad \text { for all } \tau \in \mathcal{I}, x \in \mathcal{B} .
$$

Using the results of Fukui [9, we can make the analogous definitions of $K_{m}, K_{m}^{\prime}$, and $\mathcal{B}^{\prime}$ as in Section 6. We consider the flow $J_{s, t}$ and the vector field $\hat{v}: \mathcal{I} \times \mathbb{R}^{n} \rightarrow \mathbb{R}^{n}$ as in the smooth case replacing $\varphi$ with $\kappa$. The vector field

$$
\hat{v}: \mathcal{I} \times \mathbb{R}^{n} \rightarrow \mathbb{R}^{n}
$$

is a time dependent Lipschitz vector field on $\mathbb{R}^{n}$. As in the smooth case, we consider the flow $J_{s, t}$ associated with the time dependent vector field $\hat{v}$ satisfying

$$
\frac{\partial J_{s, t}}{\partial s}(x)=\hat{v}_{s}\left(J_{s, t}(x)\right), \quad J_{t, t}(x)=x .
$$

For $\omega \in \mathfrak{D}^{r}\left(\mathbb{R}^{n}\right)$ it follows that

$$
\left.\frac{\partial\left(J_{\tau, t}^{\#} \omega\right)}{\partial \tau}\right|_{\tau=s}=J_{s, t}^{\#}\left(\mathcal{L}_{\hat{v}_{s}} \omega\right)
$$

where $\mathcal{L}_{\hat{v}_{s}} \omega$ is the Lie derivative of $\omega$ with respect to Lipschitz vector field $\hat{v}_{s}$ as discussed is Section 5. In particular,

$$
\left.\frac{\partial\left(\kappa_{\tau}^{\#} \omega\right)}{\partial \tau}\right|_{\tau=t}=\kappa_{t}^{\#}\left(\mathcal{L}_{\hat{v}_{t}} \omega\right)
$$

We note that for a given $\omega \in \mathfrak{D}^{r}\left(\mathbb{R}^{n}\right)$, the curve $t \mapsto \kappa_{t}^{\#} \omega$ is valued in the space of flat $r$-forms in $\mathcal{B}$. As in section 6 , the curve $t \mapsto \kappa_{t \#}(T)$ is used to model the time evolution of the generalized domain. The main results described in Section 7 apply in the case of Lipschitz motions.

Lemma 8.1. For the mapping $\kappa$, as defined in Equation (8.2), and a flat chain $T \in F_{r, K_{m}}(\mathcal{B})$ with $M(T)<\infty$, the curve $t \mapsto \kappa_{t \#}(T)$, where $\kappa_{t \#}(T)$ is defined in Section 4 by $\kappa_{t \#}(T)=\lim _{\rho \rightarrow 0}\left(\Phi_{\rho} * \kappa_{t}\right)(T)$, is continuous with respect to the $M_{K_{m}^{\prime}}$-norm on $\mathfrak{D}_{r}\left(\mathbb{R}^{n}\right)$. 
Proof. let $t \in \mathcal{I}$ and select $\varepsilon$ such that $t+\varepsilon \in \mathcal{I}$. Then,

$$
\begin{aligned}
& M_{K_{m}^{\prime}}\left(\kappa_{t+\varepsilon \#} T-\kappa_{t \#} T\right) \\
& \quad= \sup _{\omega \in \mathfrak{D}^{r}\left(\mathbb{R}^{n}\right)}\left\{\lim _{\rho \rightarrow 0} \frac{T\left(\left(\Phi_{\rho} * \kappa_{t+\varepsilon}\right)^{\#} \omega-\left(\Phi_{\rho} * \kappa_{t}\right)^{\#} \omega\right)}{M_{K_{m}^{\prime}}(\omega)}\right\}, \\
& \quad \leq \sup _{\omega \in \mathfrak{D}^{r}\left(\mathbb{R}^{n}\right)}\left\{\lim _{\rho \rightarrow 0} \frac{M(T) M_{K_{m}}\left(\left(\Phi_{\rho} * \kappa_{t+\varepsilon}\right)^{\#} \omega-\left(\Phi_{\rho} * \kappa_{t}\right)^{\#} \omega\right)}{M_{K_{m}^{\prime}}(\omega)}\right\},
\end{aligned}
$$

and as in the proof of Lemma 7.2

$$
\begin{aligned}
& M_{K_{m}}\left(\left(\Phi_{\rho} * \kappa_{t+\varepsilon}\right)^{\#} \omega-\left(\Phi_{\rho} * \kappa_{t}\right)^{\#} \omega\right) \\
& \leq M_{K_{m}^{\prime}}(\omega)\left(\mathfrak{L}_{\left(\Phi_{\rho} * \kappa_{t+\varepsilon}-\Phi_{\rho} * \kappa_{t}\right), K_{m}}\right)^{r} \\
&+\left(\mathfrak{L}_{\Phi_{\rho} * \kappa_{t}, K_{m}}\right)^{r} M_{K_{m}^{\prime}}\left(\omega \circ\left(\Phi_{\rho} * \kappa_{t+\varepsilon}\right)-\omega \circ\left(\Phi_{\rho} * \kappa_{t}\right)\right) .
\end{aligned}
$$

In addition,

$$
\lim _{\rho \rightarrow 0} \mathfrak{L}_{\left(\Phi_{\rho} * \kappa_{t+\varepsilon}-\Phi_{\rho} * \kappa_{t}\right), K_{m}}=\lim _{\rho \rightarrow 0} \mathfrak{L}_{\left(\Phi_{\rho} *\left(\kappa_{t+\varepsilon}-\kappa_{t}\right)\right), K_{m}}=\mathfrak{L}_{\kappa_{t+\varepsilon}-\kappa_{t}, K_{m}} .
$$

We now consider the limit of the foregoing estimate when $\varepsilon \rightarrow 0$. By the continuity of the motion it follows that $\mathfrak{L}_{\kappa_{t+\varepsilon}-\kappa_{t}, K_{m}} \rightarrow 0$ as $\varepsilon \rightarrow 0$. The second term in the estimate above vanishes by the continuity of $\omega$ and the continuity of the mollified motion $\Phi_{\rho} * \kappa$ with respect to the strong Lipschitz topology. In conclusion,

$$
\lim _{\varepsilon \rightarrow 0} M_{K_{m}^{\prime}}\left(\kappa_{t+\varepsilon \#} T-\kappa_{t \#} T\right)=0 .
$$

The following theorem is a generalization of Theorem 7.3

Theorem 8.2. Let $\kappa$ be a map associated with a motion as defined by Equation (8.2) and let $T \in F_{r, K_{m}}(\mathcal{B})$ be a flat chain of finite mass. The derivative $\left.\frac{d}{d t}\left(\kappa_{t \#} T\right)\right|_{t=\tau}$, exists in the topology of $S_{K_{m}^{\prime}}\left(\mathbb{R}^{n}\right)$ and is given by

$$
\left.\frac{d}{d t}\left(\kappa_{t \#} T\right)\right|_{t=\tau}=\partial\left(\hat{v}_{\tau} \wedge \kappa_{\tau \#} T\right)+\hat{v}_{\tau} \wedge \partial\left(\kappa_{\tau \#} T\right) .
$$

Proof. We have to show that

$$
\lim _{\varepsilon \rightarrow 0} S_{K_{m}^{\prime}}\left(\frac{\kappa_{\tau+\varepsilon \#} T-\kappa_{\tau \#} T}{\varepsilon}-\left(\partial\left(\hat{v}_{\tau} \wedge \kappa_{\tau \#} T\right)+\hat{v}_{\tau} \wedge \partial\left(\kappa_{\tau \#} T\right)\right)\right)=0 .
$$

In an analogous manner to the proof of theorem 7.3 , and as the homotopy theorem holds for the Lipschitz case, it follows that 


$$
\begin{aligned}
& \frac{1}{\varepsilon} S_{K_{m}^{\prime}}\left(\varphi_{t+\varepsilon \#} T-\varphi_{t \#} T-\varepsilon\left(\partial\left(\hat{v}_{t} \wedge \varphi_{t \#} T\right)+\hat{v}_{t} \wedge \partial\left(\varphi_{t \#} T\right)\right)\right) \\
& \leq \frac{1}{\varepsilon} \sup _{\omega \in \mathfrak{D}^{r}\left(\mathbb{R}^{n}\right)}\left\{\frac{\int_{t}^{t+\varepsilon}\left[T\left(\varphi_{\tau}^{\#} \mathcal{L}_{\hat{v}_{\tau}} \omega\right)\right] d L_{\tau}^{1}-\varepsilon T\left(\left(\varphi_{t}^{\#} \mathcal{L}_{\hat{v}_{t}} \omega\right)\right)}{S_{K_{m}^{\prime}}(\omega)}\right\}, \\
& \leq \sup _{\omega \in \mathfrak{D}^{r}\left(\mathbb{R}^{n}\right)}\left\{M(T) \sup _{s \in[t, t+\varepsilon]} \frac{M_{K_{m}}\left(\varphi_{s}^{\#} \mathcal{L}_{\hat{v}_{s}} \omega-\varphi_{t}^{\#} \mathcal{L}_{\hat{v}_{t}} \omega\right)}{S_{K_{m}^{\prime}}(\omega)}\right\} .
\end{aligned}
$$

The term $M_{K_{m}}\left(\varphi_{s}^{\#} \mathcal{L}_{\hat{v}_{s}} \omega-\varphi_{t}^{\#} \mathcal{L}_{\hat{v}_{t}} \omega\right)$ may be estimated as

$$
\begin{aligned}
M_{K_{m}}\left(\varphi_{s}^{\#} \mathcal{L}_{\hat{v}_{s}} \omega-\varphi_{t}^{\#} \mathcal{L}_{\hat{v}_{t}} \omega\right) \\
\quad \leq M_{K_{m}}\left(\varphi_{s}^{\#} \mathcal{L}_{\hat{v}_{s}} \omega-\varphi_{s}^{\#} \mathcal{L}_{\hat{v}_{t}} \omega+\varphi_{s}^{\#} \mathcal{L}_{\hat{v}_{t}} \omega-\varphi_{t}^{\#} \mathcal{L}_{\hat{v}_{t}} \omega\right) \\
\quad \leq M_{K_{m}}\left(\varphi_{s}^{\#} \mathcal{L}_{\hat{v}_{s}} \omega-\varphi_{s}^{\#} \mathcal{L}_{\hat{v}_{t}} \omega\right)+M_{K_{m}}\left(\varphi_{s}^{\#} \mathcal{L}_{\hat{v}_{t}} \omega-\varphi_{t}^{\#} \mathcal{L}_{\hat{v}_{t}} \omega\right), \\
\quad \leq M_{K_{m}}\left(\varphi_{s}^{\#}\left(\mathcal{L}_{\hat{v}_{s}-\hat{v}_{t}} \omega\right)\right)+M_{K_{m}}\left(\varphi_{s}^{\#} \mathcal{L}_{\hat{v}_{t}} \omega-\varphi_{t}^{\#} \mathcal{L}_{\hat{v}_{t}} \omega\right) .
\end{aligned}
$$

By Equation (5.7) we have

$$
\begin{aligned}
M_{K_{m}}\left(\varphi_{s}^{\#}\left(\mathcal{L}_{\hat{v}_{s}-\hat{v}_{t}} \omega\right)\right) & \leq\left(\mathfrak{L}_{\varphi_{s}, K_{m}}\right)^{r} M_{K_{m}^{\prime}}\left(\mathcal{L}_{\hat{v}_{s}-\hat{v}_{t}} \omega\right) \\
& \leq\left(\mathfrak{L}_{\varphi_{s}, K_{m}}\right)^{r} C(n, r) S_{K_{m}^{\prime}}(\omega)\left\|\hat{v}_{s}-\hat{v}_{t}\right\|_{\mathfrak{L}, K_{m}^{\prime}}
\end{aligned}
$$

and since $\left\|\hat{v}_{s}-\hat{v}_{t}\right\|_{\mathfrak{L}, K_{m}^{\prime}} \rightarrow 0$ as $s \rightarrow t$, it follows that

$$
\lim _{s \rightarrow t} M_{K_{m}}\left(\varphi_{s}^{\#}\left(\mathcal{L}_{\hat{v}_{s}-\hat{v}_{t}} \omega\right)\right)=0 .
$$

Lemma 8.1, implies that

$$
\lim _{s \rightarrow t} M_{K_{m}}\left(\varphi_{s}^{\#} \mathcal{L}_{\hat{v}_{t}} \omega-\varphi_{t}^{\#} \mathcal{L}_{\hat{v}_{t}} \omega\right)=0
$$

which completes the proof.

\section{The Product Rule and the Transport Theorem}

In this section we apply the foregoing results to obtain a generalized formulation of the transport theorem for a region of any dimension, and in particular, the surface transport theorem. In view of the postulates for a motion described in Section 8, we recall that for an $r$-current of finite mass, $T \in F_{r, K_{m}}(\mathcal{B})$, and the map $\kappa: \mathcal{I} \times \mathcal{B} \rightarrow \mathbb{R}^{n}$ associated with a motion by Equation (8.2), one has

$$
\kappa_{t \#}(T) \in F_{r, K_{m}^{\prime}}\left(\mathbb{R}^{n}\right), \quad \text { and } \quad \frac{d}{d t}\left(\kappa_{t \#}(T)\right) \in S_{r, K_{m}^{\prime}}\left(\mathbb{R}^{n}\right), \quad \text { for all } t \in \mathcal{I} \text {. }
$$


The standard transport theorem is concerned with the integration of a density of some extensive property over an evolving region. Here, the evolving region is generalized and is represented by the evolving flat chain $\kappa_{t \#}(T)$, and the extensive property at any instant is represented by a sharp cochain. As any sharp cochain is a flat cochain, the integration operation in the classical formulation of the theorem is replaced by the action of a cochain on a chain. Thus, an evolving extensive property, $\psi$, is viewed as a continuous curve in the space of sharp $r$-cochains, that is, a continuous mapping

$$
X_{\psi}: \mathcal{I} \rightarrow\left[S_{r}\left(\mathbb{R}^{n}\right)\right]^{*} .
$$

Moreover, we assume that the curve is differentiable in the topology of flat $r$-cochains in $\mathbb{R}^{n}$. That is, the limit

$$
\lim _{\varepsilon \rightarrow 0} \frac{X_{\psi}(\tau+\varepsilon)-X_{\psi}(\tau)}{\varepsilon},
$$

exists as a flat $r$-cochain, and it will denoted by $\dot{X}_{\psi}(\tau)$.

For each $t \in \mathcal{I}$ the total of the extensive property in the flat $r$-chain $T$ is therefore

$$
X_{\psi}\left(\kappa_{\#}(T)\right)(t)=X_{\psi}(t)\left(\kappa_{t \#}(T)\right) .
$$

For the time derivative of $X_{\psi}\left(\kappa_{\#}(T)\right)$, it easily follows that we have

$$
\begin{aligned}
\frac{d}{d t} X_{\psi}\left(\kappa_{\#}(T)\right) & =\lim _{\varepsilon \rightarrow 0} \frac{X_{\psi}(t+\varepsilon)\left(\kappa_{t+\epsilon \#}(T)\right)-X_{\psi}(t)\left(\kappa_{t \#}(T)\right)}{\varepsilon}, \\
& =X_{\psi}(t)\left(\frac{d}{d t} \kappa_{t \#}(T)\right)+\frac{d X_{\psi}}{d t}(t)\left(\kappa_{t \#}(T)\right) .
\end{aligned}
$$

By Equation (8.4), it follows that

$$
\begin{aligned}
& \left.\frac{d}{d t}\left(X_{\psi}\left(\kappa_{\#}(T)\right)\right)\right|_{t=\tau} \\
& =\dot{X}_{\psi}(\tau)\left(\kappa_{\tau \#}(T)\right)+X_{\psi}(\tau)\left(\partial\left(\hat{v}_{\tau} \wedge \kappa_{\tau \#}(T)\right)+\hat{v}_{\tau} \wedge \kappa_{\tau \#}(\partial T)\right) .
\end{aligned}
$$

In the case where $X_{\psi}$ is represented by a smooth differential $r$-form $D_{\psi}$ and $\kappa$ is a smooth embedding we obtain

$$
\begin{aligned}
\left.\frac{d}{d t}\left(X_{\psi}(t)\left(\kappa_{t \#}(T)\right)\right)\right|_{t=\tau} & =\left(\kappa_{\tau \#}(T)\right)\left(\dot{D_{\psi}}+d D_{\psi}\left\llcorner\hat{v}_{\tau}+d\left(D_{\psi}\left\llcorner\hat{v}_{\tau}\right)\right)\right.\right. \\
& =\kappa_{\tau \#}(T)\left(\dot{D_{\psi}}+\mathcal{L}_{\hat{v}_{\tau}} D_{\psi}\right)
\end{aligned}
$$

which is a generalization of [3, equation (T0)]. For a time dependent $r$-form $D_{\psi}$, using [1, pp. 367] and the notation of Section 8, it follows that

$$
\left.\frac{d}{d t} \kappa_{t}^{\#}\left(D_{\psi}(t)\right)\right|_{t=\tau}=\kappa_{\tau}^{\#}\left(\dot{D_{\psi}}(\tau)+d D_{\psi}(\tau)\left\llcorner\hat{v}_{\tau}+d\left(D_{\psi}(\tau)\left\llcorner\hat{v}_{\tau}\right)\right) .\right.\right.
$$

Applying the last expression to Equation (9.1), we obtain the following relation between the Eulerian and Lagrangian formulations of the transport 
theorem

$$
\left.\frac{d}{d t}\left(X_{\psi}\left(\kappa_{\#}(T)\right)(t)\right)\right|_{t=\tau}=\left(\left.\frac{d}{d t} \kappa_{t}^{\#}\left(X_{\psi}(t)\right)\right|_{t=\tau}\right) T .
$$

For the general case, the sharp $r$-cochain $X_{\psi}(\tau)$ is represented by a sharp $r$-form $D_{\psi}(\tau)$ and the sharp $r$-cochain $\dot{X}_{\psi}(\tau)$ is represented by the flat $r$ form $\dot{D}_{\psi}(\tau)$. As $T$ is a flat chain of finite mass it may be represented by $L^{n}$-integrable vector field $T=\eta \wedge L^{n}$. It is concluded that

$$
\begin{aligned}
\frac{d}{d t}\left(X_{\psi}\left(\kappa_{\#}(T)\right)\right) & \left.\right|_{t=\tau} \\
= & \left(\kappa_{\tau \#}(T)\right)\left(\dot{D}_{\psi}(\tau)+d D_{\psi}(\tau)\left\llcorner\hat{v}_{\tau}+d\left(D_{\psi}(\tau)\left\llcorner\hat{v}_{\tau}\right)\right),\right.\right. \\
& =\int_{\mathcal{B}} \kappa_{\tau}^{\#}\left(\dot{D}_{\psi}(\tau)+d D_{\psi}(\tau)\left\llcorner\hat{v}_{\tau}+d\left(D_{\psi}(\tau)\left\llcorner\hat{v}_{\tau}\right)\right)(\eta) d L_{x}^{n} .\right.\right.
\end{aligned}
$$

As an example consider the situation where the flat form $D_{\psi}$ satisfies Cauchy's postulates. Then, associated with the property are a time dependent flat $r$-form $D_{\phi}$ representing the source, and a time dependent flat $(r-1)$-form $D_{\xi}$ representing the flux, such that the differential balance equation for the property is (see [26])

$$
\dot{D_{\psi}}+d D_{\xi}=D_{\phi}
$$

Each of the forms above represents a flat cochain denoted by $X_{\omega}, X_{\xi}, X_{\phi}$, respectively, such that

$$
\dot{X}_{\omega}+d X_{\xi}=X_{\phi}
$$

Thus, the transport formula assumes the form

$$
\begin{aligned}
& \left.\frac{d}{d t}\left(X_{\psi}\left(\kappa_{\#}(T)\right)\right)\right|_{t=\tau} \\
= & \dot{X}_{\psi}(\tau)\left(\kappa_{\tau \#}(T)\right)+X_{\psi}(\tau)\left(\left.\frac{\partial}{\partial t} \kappa_{t \#}(T)\right|_{t=\tau}\right), \\
= & \dot{X}_{\psi}(\tau)\left(\kappa_{\tau \#}(T)\right)+X_{\psi}(\tau)\left(\partial\left(\hat{v}_{\tau} \wedge \kappa_{\tau \#} T\right)+\hat{v}_{\tau} \wedge \partial\left(\kappa_{\tau \#} T\right)\right), \\
= & \left(X_{\phi}\right)\left(\kappa_{\#}(T)\right)(\tau)+\left(X_{\psi}\left\llcorner\hat{v}-X_{\xi}\right)\left(\kappa_{\#}(\partial T)\right)(\tau)+X_{\psi}\left(\partial\left(\hat{v} \wedge \kappa_{\#} T\right)\right)(\tau) .\right.
\end{aligned}
$$

Acknowledgments. This work was partially supported by the Perlstone Center for Aeronautical Engineering Studies, the Kreitman Post-Doctoral Scholarship and the H. Greenhill Chair for Theoretical and Applied Mechanics at Ben-Gurion University of the Negev. The authors wish to thank M. Silhavy for the discussions and comments he has made on earlier versions of this work. 


\section{REFERENCES}

[1] R. Abraham, J. E. Marsden, and T. Ratiu. Manifolds, Tensor Analysis, and Applications. Springer, 1988.

[2] S. Angenent and M.E. Gurtin. Multiphase thermomechanics with interfacial structure 2. evolution of an isothermal interface. Archive for Rational Mechanics and Analysis, 108:323-391, 1989.

[3] D.E. Betounes. Kinematics of submanifolds and the mean curvature normal. Archive for Rational Mechanics and Analysis, 96:1-27, 1986.

[4] F. Bouchut and G. Crippa. Uniqueness, renormalization and smooth approximations for linear transport equations. SIAM J. Math. Anal., 4:1316-1328, 2006.

[5] K.P. Chi, N.H. Quang, and B.C. Van. The Lie derivative of currents on Lie groups. Lobachevskii Journal of Mathematics, 33(1):10-21, 2012.

[6] L. Falach and R. Segev. The configuration space and principle of virtual power for rough bodies. Mathematics and Mechanics of Solids, 2013.

[7] L. Falach and R. Segev. Reynolds transport theorem for smooth deformations of currents on manifolds. Accepted to Mathematics and Mechanics of Solids, DOI: 10.1177/1081286514551503, 2014.

[8] H. Federer. Geometric Measure Theory. Springer, 1969.

[9] K. Fukui and T. Nakamura. A topological property of Lipschitz mappings. Topology and its Applications, 148:143-152, 2005.

[10] M. Giaquinta, G. Modica, and J. Soucek. Cartesian Currents in the Calculus of Variation I. Springer, 1998.

[11] M. Golubitsky and V. Guillemin. Stable Mappings and Their Singularities. Springer, 1973.

[12] M.E. Gurtin. Multiphase thermomechanics with interfacial structure 1. heat conduction and the capillary balance law. Archive for Rational Mechanics and Analysis, 104:195-221, 1988.

[13] M.E. Gurtin. Configurational Forces as Basic Concepts of Continuum Physics. Springer, 2000.

[14] M.E. Gurtin and M.E. Jabbour. Interface evolution in three dimentions with curvature-dependent energy and surface diffusion: Interface-controlled evolution, phase transition, epitaxial growth of elastic films. Archive for Rational Mechanics and Analysis, 163:171-208, 2002.

[15] M.E. Gurtin and A.I. Murduch. A continuum theory of elastic material surfaces. Archive for Rational Mechanics and Analysis, 57:291-323, 1975.

[16] M.E. Gurtin and A. Struthers. Multiphase thermodynamics with interfacial structure 3. evolving phase boundaries in the presence of bulk deformation. Archive for Rational Mechanics and Analysis, 112:97-160, 1990.

[17] J. Harrison. Operator calculus of differential chains and differential forms. Journal of Geometric Analysis, 25:357-420, 2015.

[18] J. Harrison and H. Pugh. Topological aspects of differential chains. Journal of Geometric Analysis, 22 (3):685-690, 2012.

[19] J. Heinonen. Lectures on Analysis on Metric Spaces. Springer, 2000.

[20] M.W. Hirsch. Differential Topology. Springer, 1976.

[21] S. Lang. Fundamentals of Differential Geometry. Springer, 1999.

[22] A. Struthers M.E. Gurtin and W.O. Williams. A transport theorem for moving interfaces. Quarterly of Applied Mathematics, 47:773-777, 1989.

[23] P.W. Michor. Manifolds of Differentiable Mappings. Shiva, 1980.

[24] W. Noll and E.G. Virga. Fit regions and functions of bounded variation. Archive for Rrational Mechanics and Analysis, 102:1-21, 1988.

[25] O. Reynolds. The sub-mechanics of the universe. In Papers on Mechanical and Physical Subjects, volume III. Cambridge University Press, 1903. 
[26] R. Segev. Notes on metric independent analysis of classical fields. Mathematical Methods in the Applied Sciences, 36:497-566, 2013.

[27] B. Seguin and E. Fried. Roughening it - evolving irregular domains and transport theorem. Mathematical Methods and Methods in Applied Sciences, 24:,1729-1779, 2014.

[28] B. Seguin, D.F. Hinz, and E. Fried. Extending the transport theorem to rough domains of integration. Applied Mechanical Reviews, 66(5):050802, 2014.

[29] C.A. Truesdell and R. Toupin. The classical field theories. In S. Flügge, editor, Handbuch der Physik, volume III/1. Springer, 1960.

[30] H. Whitney. Geometric Integration Theory. Princeton University Press, 1957. 